\title{
The novel Arabidopsis thaliana svt2 suppressor of the ascorbic acid-deficient mutant vtc1-1 exhibits phenotypic and
}

\section{genotypic instability [version 1; peer review: 2 approved, 1 not}

\section{approved]}

\author{
Chase F Kempinski1,2, Samuel V Crowell ${ }^{1,3}$, Caleb Smeeth ${ }^{1,4}$, Carina Barth ${ }^{1,5}$ \\ ${ }^{1}$ Department of Biology, West Virginia University, Morgantown, 26506, USA \\ ${ }^{2}$ Department of Plant and Soil Sciences, University of Kentucky, Lexington, 40546, USA \\ ${ }^{3}$ Department of Plant Biology, Cornell University, Ithaca, 14853, USA \\ ${ }^{4}$ ACTION-Housing Inc., Pittsburgh, 15219, USA \\ ${ }^{5}$ ConRuhr North America, New York, 10017, USA
}

V1 First published: 10 Jan 2013, 2:6

https://doi.org/10.12688/f1000research.2-6.v1

Latest published: 10 Jan 2013, 2:6

https://doi.org/10.12688/f1000research.2-6.v1

\section{Abstract}

Ascorbic acid is a potent antioxidant that detoxifies reactive oxygen species when plants are exposed to unfavorable environmental conditions. In addition to its antioxidant properties, ascorbic acid and its biosynthetic precursors fulfill a variety of other physiological and molecular functions. A mutation in the ascorbic acid biosynthesis gene VTC1, which encodes GDP-mannose pyrophosphorylase, results in conditional root growth inhibition in the presence of ammonium. To isolate suppressors of vtc1-1, which is in the Arabidopsis Columbia-0 background, seeds of the mutant were subjected to ethyl methanesulfonate mutagenesis. A suppressor mutant of vtc1-12, svt2, with wild-type levels of ascorbic acid and root growth similar to the wild type in the presence of ammonium was isolated. Interestingly, svt2 has Arabidopsis Landsberg erecta features, although svt2 is delayed in flowering and has an enlarged morphology. Moreover, the svt2 genotype shares similarities with Ler polymorphism markers and sequences, despite the fact that the mutant derived from mutagenesis of Col-0 vtc1-1 seed. We provide evidence that svt2 is not an artifact of the experiment, a contamination of Ler seed, or a result of outcrossing of the svt2 mutant with Ler pollen. Instead, our results show that svt2 exhibits transgenerational genotypic and phenotypic instability, which is manifested in a fraction of svt2 progeny, producing revertants that have Col-like phenotypic and genotypic characteristics. Some of those Col-like revertants then revert back to svt2-like plants in the subsequent generation. Our findings have important implications for undiscovered phenomena in transmitting genetic information in addition to the Mendelian laws of inheritance. Our results suggest

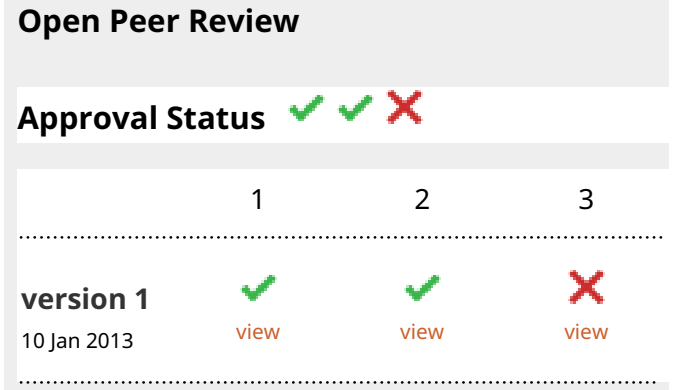

1. Andy Pereira (D), University of Arkansas, Fayetteville, AR, USA

2. Igor Kovalchuk, University of Lethbridge, Lethbridge, $A B$, Canada

3. David Oppenheimer, University of Florida,

Gainesville, FL, USA

Any reports and responses or comments on the article can be found at the end of the article. 
that stress can trigger a genome restoration mechanism that could be advantageous for plants to survive environmental changes for which the ancestral genes were better adapted.

\section{Associated Research Article}

Hopkins MT, Khalid AM, Chang PC et al. » De novo genetic variation revealed in somatic sectors of single Arabidopsis plants, F1000Research 2013, 2:5 (https://doi.org/10.12688/f1000research.2-5.v2)

Corresponding author: Carina Barth (barth@conruhr.org)

Competing interests: No relevant competing interests disclosed.

Grant information: This work was supported by a start-up grant of West Virginia University to CB. The funders had no role in study design, data collection and analysis, decision to publish, or preparation of the manuscript.

Copyright: ( $) 2013$ Kempinski CF et al. This is an open access article distributed under the terms of the Creative Commons Attribution License, which permits unrestricted use, distribution, and reproduction in any medium, provided the original work is properly cited. Data associated with the article are available under the terms of the Creative Commons Zero "No rights reserved" data waiver (CC0 1.0 Public domain dedication).

How to cite this article: Kempinski CF, Crowell SV, Smeeth C and Barth C. The novel Arabidopsis thaliana svt2 suppressor of the ascorbic acid-deficient mutant vtc1-1 exhibits phenotypic and genotypic instability [version 1; peer review: 2 approved, 1 not approved] F1000Research 2013, 2:6 https://doi.org/10.12688/f1000research.2-6.v1

First published: 10 Jan 2013, 2:6 https://doi.org/10.12688/f1000research.2-6.v1 


\section{Introduction}

L-ascorbic acid (AA, vitamin $\mathrm{C}$ ) is an important antioxidant withmultiple functions in many species. It serves as a scavenger of reactive oxygen species generated under adverse environmental conditions. However, AA also influences flowering time and senescence $^{1-3}$, pathogen disease resistance ${ }^{2,4}$, the biosynthesis of various plant hormones ${ }^{5-7}$, and root development ${ }^{8-11}$. This suggests that AA and some of its intermediates have functions in addition to its antioxidant properties.

Ascorbic acid biosynthesis in plants occurs predominantly through the D-mannose/L-galactose pathway ${ }^{12,13}$. Given the multifaceted functions of AA in plants, there is a need to advance our understanding of how plants regulate the biosynthesis and accumulation of AA. Arabidopsis thaliana mutants deficient in AA have provided important insights into the breadth of molecular and physiological functions of AA. One of the Arabidopsis mutants, vtc1-1, contains a defect in the AA biosynthetic enzyme GDP-mannose pyrophosphorylase. The mutant was originally generated by ethyl methanesulfonate (EMS) mutagenesis of Col-0 wild-type seed ${ }^{14}$. The $v t c 1-1$ mutant contains a point mutation in amino acid 22 that converts a conserved proline into a serine ${ }^{15}$. The VTCl gene has recently been shown to be a determinant of ammonium sensitivity in plants. In the presence of ammonium, vtc1-1 mutants exhibit strongly reduced root growth in comparison to the wild type, a phenomenon that is independent of AA deficiency ${ }^{8-11}$. To better understand the mechanism through which VTC1 mediates conditional ammonium sensitivity, it is important to identify regulatory partners of VTC1. To accomplish this, we undertook a suppressor mutagenesis approach of vtc1-1 homozygous mutant seed in the hope of identifying vtcl-1 suppressor mutants that could then be isolated and studied.

One of the suppressor mutants isolated in the $\mathrm{M}_{0}$ generation, svt2 (suppressor of vtc1-1 2), contained wild-type AA levels and developed roots similar to the wild type in the presence of ammonium. However, while characterizing the mutant genotypically, we observed that it lost the original vtcl-1 mutation (i.e., svt 2 contained the homozygous wild-type allele). Furthermore, we determined that svt2, although generated through EMS mutagenesis of Col-0 vtc1-1 mutant seed, was phenotypically and genotypically similar to Ler. Intriguingly, a small percentage of $s v t 2 \mathrm{M}_{1}$ plants produced offspring that have phenotypic and genotypic similarities to $\mathrm{Col}$ in the $\mathrm{M}_{2}$ generation. Even more remarkably, a small percentage of Col-like revertants in the $\mathrm{M}_{2}$ generation produced progeny that exhibited phenotypic and genotypic svt2 characteristics again in the $M_{3}$ generation.

Phenotypic instability of Arabidopsis alleles affecting a disease resistance gene cluster has recently been reported ${ }^{16}$. In their work, Yi and Richards described that exposure to EMS or through the generation of different $\mathrm{F}_{1}$ hybrids induced phenotypic instability in the bal and cprl mutant alleles. The authors later proposed that the high phenotypic instability is caused by a genetic mechanism ${ }^{17}$.

The presented study focuses on describing and characterizing the Arabidopsis svt 2 suppressor mutant and its phenotypic and genotypic behavior. After illustrating the phenotypic features of $s v t 2$, we investigate transgenerational changes in the phenome and genome of $s v t 2$ and provide evidence that $s v t 2$ is a true mutant and not the result of an experimental artifact or contamination. Finally, we discuss our experimental findings in respect to the vtcl-1 mutant background and other reports that previously described similar phenomena of genome instability and restoration, and we briefly speculate on possible mechanisms of phenome and genome instability in $s v t 2$.

\section{Materials and methods}

Plant material and growth conditions

Arabidopsis thaliana Col-0 wild type and the previously described vtcl-1 mutant ${ }^{14}$ (in the Col-0 background) were kindly provided by Patricia Conklin (SUNY Cortland, NY, USA). Ler-0 wild-type seed were obtained from The Arabidopsis Biological Resource Center (www.arabidopsis.org). Plants were grown in Metromix 360 potting soil at $23^{\circ} \mathrm{C}$ at both day and night with a 16-hour photoperiod at $160 \mu \mathrm{mol}$ photons $\mathrm{m}^{-2} \mathrm{~s}^{-2}$ (fluorescent bulbs).

For assessment of root growth, seed of the wild types and mutant lines were surface-sterilized (see below) and grown on basal full strength $1 \times$ Murashige and Skoog (MS) medium without vitamins (Cat.\# MSP01, Caisson Laboratories, Inc., North Logan, UT), containing 1\% Phytoblend (Cat.\# PTP01, Caisson Laboratories) in omni trays (Fisher Scientific, Pittsburgh, PA) as described ${ }^{11}$. Sucrose was omitted from the tissue culture medium. The $\mathrm{pH}$ of the medium was adjusted with $\mathrm{KOH}$ to 5.7. Trays were sealed with two layers of 3M micropore tape (Fisher Scientific), put in vertical orientation, and placed in the growth chamber under long days $(16 \mathrm{~h}$ light, $8 \mathrm{~h}$ dark) at $23^{\circ} \mathrm{C}$ day and night, and $160 \mu \mathrm{mol}$ photons $\mathrm{m}^{-2} \mathrm{~s}^{-1}$ in a growth chamber (Percival Scientific, Inc., Perry, IA). Each plate contained wild-type and mutant seed. Primary root length was measured in seven-day-old seedlings using a ruler.

To assess AA content in leaf tissue, seeds of wild type and mutants were randomly sown on MetroMix 360 soil (BFG supplies Co., Burton, $\mathrm{OH}$ ) in the same flat under the growth conditions described above. When plants were three weeks old, whole rosettes were harvested for the AA assay.

\section{Seed-surface sterilization}

Seeds were soaked for $1 \mathrm{~min}$ in 50\% ethanol, followed by washing the seeds in $50 \%$ bleach plus $0.01 \%$ sodium dodecyl sulphate for 6 min. Finally, seeds were rinsed six times with sterile water and stored in $0.1 \%$ sterile Phytoblend agar for $2 \mathrm{~d}$ at $4{ }^{\circ} \mathrm{C}^{18}$.

\section{Ethyl methanesulfonate mutagenesis}

Seeds of homozygous vtc1-1 Arabidopsis thaliana (Col-0 background) were mutagenized with $0.2 \%$ ethyl methanesulfonate as described (Figure $1 ;{ }^{18}$ ). Approximately, $1200 \mathrm{M}_{0}$ seed were stratified for 4 days at $4^{\circ} \mathrm{C}$ in $0.1 \%$ agar, sown on MetroMix soil and grown as above. Plants were screened for wild-type AA levels using the nitroblue tetrazolium assay ${ }^{19}$. Additional suppressor mutants were isolated by pooling seeds generated from $\mathrm{M}_{1}$ plants. Putative mutants were isolated and allowed to self-pollinate to obtain seed.

\section{Pollen grain analysis and microscopy}

Pollen was taken from 4.5-week-old flowering plants of Col-0 and Ler wild type and vtcl-1 and svt $2 \mathrm{M}_{2}$ mutants, mounted in glycerol, and photographed using bright field settings on a Nikon E800 microscope equipped with a CoolSNAP cf CCD camera (Photometrics, Tuscon, AZ, USA). 


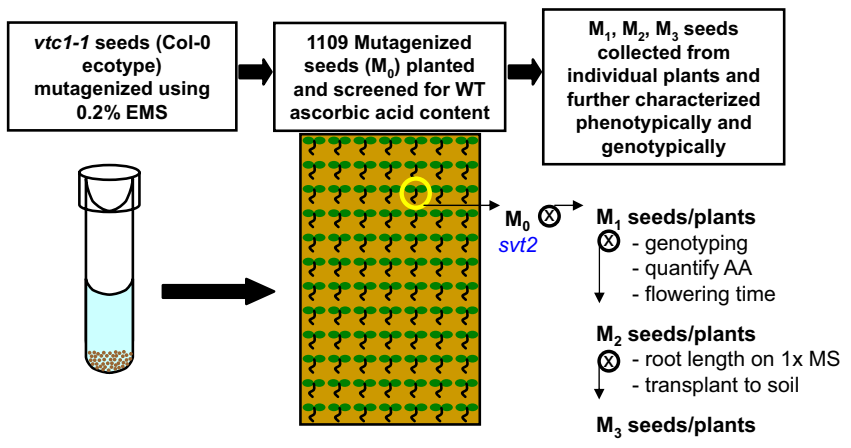

Figure 1. Isolation of svt2. To isolate vtc1-1 suppressor mutants, homozygous vtc1-1 seed (in the Col-0 genetic background) were exposed to chemical mutagenesis using ethyl methanesulfonate (EMS). Over 1000 mutagenized seed $\left(M_{0}\right)$ were planted on soil and screened for wild-type levels of ascorbic acid. The only mutant isolated in the $M_{0}$ generation containing recovered ascorbic acid levels was svt2. The mutant was allowed to self-fertilize and was characterized phenotypically and genotypically in subsequent generations.

\section{Genomic DNA isolation}

Genomic DNA was isolated from rosette leaves following a previously described $\operatorname{protocol}^{3}$. In case of genomic DNA isolation from vtcl-1 seeds, a small amount of dried seeds was crushed and the extraction procedure described previously ${ }^{3}$ was followed. Primers for the VTC1 gene and for the Insertion/Deletion (InDel) polymorphisms were designed using sequence data available on The Arabidopsis Information Resource (TAIR) database (www.arabidopsis.org). Polymerase chain reaction (PCR) was used to amplify fragments of the VTC1 gene for sequencing and to assess InDel polymorphisms. Sequences of primers used for sequencing and InDel analysis are summarized in Table 1. PCR reactions were run on $1.0 \%$ agarose gels stained with ethidium bromide.

\section{Gene copy analysis using qPCR}

Quantitative PCR reactions were set up to measure gene copy number using 2.5 pmole gene-specific primers, $300 \mathrm{ng}$ of genomic DNA diluted in DNase/RNase free water, and iQ SYBR Green supermix (Bio-Rad, Hercules, CA, USA) for a total volume of $10 \mu \mathrm{L}$. Reactions without template were used as negative controls. Each single copy reaction was set up in triplicate and run in a Bio-Rad iCycler for 40 cycles. Threshold cycles $\left(\mathrm{C}_{\mathrm{T}}\right)$ were calculated using iQ software (Bio-Rad).

Primer efficiencies (E) were calculated using cDNAs synthesized from RNA isolated from Col-0 plants as previously described ${ }^{11}$. cDNA samples were serially diluted across three orders of magnitude. Serial dilutions were amplified in triplicate using the same protocol as for the copy number experiment. The $\mathrm{C}_{\mathrm{T}} \mathrm{s}$ of each triplicate were averaged and plotted against the dilution factor. A linear trend was fitted to the data and the slope of this trend was used to calculate $\mathrm{E}$ for each primer with the formula: $\mathrm{E}=10^{\text {(11-slope) }}$.

Copy number of VTC1 (AT2G39770) was calculated using the formula: Reported Quantity $(\mathrm{RQ})=1 / \mathrm{E}^{\mathrm{CT}}$ normalized to the RQ of a known single copy gene (PAD4, AT3G52430; ${ }^{20,21}$ ). VTC1 RQ was calculated from the average VTC1 RQ of three biological replicates per genotype and was normalized to the average RQ of PAD4 from
Table 1. Forward $(F)$ and reverse $(R)$ sequences of primers used in analyzing the VTC1 gene and for amplifying five Col/Ler Insertion/Deletion (InDel) polymorphisms.

\begin{tabular}{|c|c|}
\hline Primer Name & $5^{\prime} 3^{\prime}$ \\
\hline VTC1G1 F & AAA AAT TCG TTC TAG ATG GAT GCT \\
\hline VTC1G1 R & ATG GCT GTA AAT TGG AAG AGA T \\
\hline VTC1 G2 F & GAA CCC TTG TCT CTA AAA TA \\
\hline VTC1 G2 R & CAA ATC CCA TAA TCT GTT CC \\
\hline VTC1 G3 F & CAA TTT TGC TTA CTT CTC T \\
\hline VTC1 G3 R & TGG ATG CAA CCG ACA CAA AAC AAT \\
\hline VTC1G4 F & ACA TTT TTA GCA GCT GGT ATT GAG \\
\hline VTC1G4 R & AGG TAA GAA CTG GCA GAC TAA AG \\
\hline VTC1 G5 F & TCG CTT GAG ACC ATT GAC T \\
\hline VTC1 G5 R & GAG GCT TCC CCA CCG TGA GAT TTG \\
\hline VTC1 G6 F & CAA GCT GGA AAT CAA AAT CAC T \\
\hline VTC1 G6 R & GCG CTG CTG CAA TCT TAG G \\
\hline VTC1 G7 F & ACA AAT CTC ACG GTG GGG AAG C \\
\hline VTC1 G7 R & TGG TTA ATT TGG CAG GAG A \\
\hline VTC1 G8 F & CAA GGG CTC TAT GCT ATG GTG \\
\hline VTC1 G8 R & GCG TTT TGA TTG ATG CTT ATT C \\
\hline VTC1 G9 F & GCG TGT ATC TCG AGC AGT ATC AT \\
\hline VTC1 G9 R & GTG GAG GGA AGT TAA GGG TAT TTT \\
\hline InDel $1450919 \mathrm{~F}$ & ATC GGT TTG TAA TCT CTG TCC A \\
\hline InDel 1450919 R & TAT GCG TTC CCA AAT TTG TTA TCT C \\
\hline Indel $2451470 \mathrm{~F}$ & GGA GAC CCA AAC TGC TAT TAC A \\
\hline Indel $2451470 \mathrm{R}$ & AAC CGC CTC CAT TTG CAC CTT ATC \\
\hline Indel 3469762 F & GTC ACC GAG TTT TGC TTT GTT CAT \\
\hline Indel 3469762 R & CTC GTT TCT TTT CTG GGC TTG TAG \\
\hline Indel $4449053 \mathrm{~F}$ & GAA AGA AAG CAG CGA AAG ACA \\
\hline Indel 4449053 R & GCC CAT GCC CAT ACA CTG A \\
\hline Indel $5455100 \mathrm{~F}$ & ACT TGC TTA ATC GTT TCT TTG TA \\
\hline Indel $5455100 \mathrm{R}$ & GCC CAC TCG TAT TCG CTT AG \\
\hline
\end{tabular}

three replicates of each respective genotype, all run in the same reaction plate.

Sequencing analysis

PCR products were purified using the Qiagen Miniprep Kit. Dyeterminator based DNA sequencing was performed at the Genomics Facility in the Department of Biology at West Virginia University. Sequence alignments were performed using the BioEdit program (http://www.mbio.ncsu.edu/bioedit/bioedit.html).

\section{Ascorbic acid quantification}

To screen mutants, AA levels were analyzed qualitatively in small pieces of two-week-old rosette leaves using the nitroblue tetrazolium assay previously described ${ }^{19}$. The AA content was determined in whole rosettes of three-week-old plants using the iron reduction assay ${ }^{4}$.

Statistical analysis

Experiments were performed at least three times. Figures represent individual experiments. Data were expressed as mean values $\pm \mathrm{SE}$. $P$ values were determined by Student's $t$ test analysis. 


\section{Results}

Isolation of svt2

Our laboratory is interested in understanding how the VTC1 gene, which is essential for the biosynthesis of GDP-mannose and AA, is regulated. This would help deciphering the pleiotropic phenotypes displayed by $v t c 1-1$, including its hypersensitivity to ammonium ${ }^{8-11}$. We employed a gene suppressor analysis with the goal of identifying novel genes that interact or regulate VTC1. Seed of the vtcl-1 mutant, which is in the Col-0 genetic background ${ }^{14}$, were subjected to chemical mutagenesis using $\mathrm{EMS}^{18}$. One thousand and one hundred mutagenized $v t c 1-1$ seeds $\left(\mathrm{M}_{0}\right.$ generation) were planted onto soil and screened for recovered (wild-type) leaf AA content using the qualitative nitroblue tetrazolium test ${ }^{19}$. One of the mutants exhibited wild-type AA levels in the $\mathrm{M}_{0}$ generation. This mutant was named svt2 (suppressor of vtc1-1 2), isolated, and further characterized. The mutant was allowed to self-fertilize and seeds from the plant were collected ( $\mathbf{M}_{1}$ generation) (Figure 1). Note that we isolated additional suppressor mutants by pooling $\mathrm{M}_{2}$ seed and by screening for long roots on $1 \times$ Murashige and Skoog (MS) medium containing ammonium. Six suppressor mutants were identified among 2000 plants. $M_{3}$ seed were collected and screened for long roots again to test for segregation. $\mathrm{M}_{4}$ progeny of one line had all long roots, whereas the other five lines segregated in a ratio of three plants producing long roots, and one plant having short roots. Figure 2 summarizes data of four of these suppressor mutants, with D3-4 homogenously producing long roots, whereas D3-3, D3-7, and D3-15 developed long and short roots in a 3:1 ratio. As is illustrated in Figure 2A, these suppressor mutants developed roots that were significantly longer than those of the Col-0 wild type. Analysis of the total AA content revealed that the suppressor D3-4 had an AA content comparable to the Col-0 wild type, whereas that of $v t c 1-1$ was only approximately $40 \%$ of that of the wild type ${ }^{14,15}$.
Finally, sequence analysis of these four suppressor mutants demonstrated a lack of the $v t c 1-1$ mutation (Figure 2C). Except for the assessments described above, these suppressor mutants were not yet characterized further.

$\begin{aligned} & \text { Root lengths of Col-0 WT, vtc1-1, and D3 suppressor mutants } \\ & (\mathbf{m m})\end{aligned}$
5 Data Files
http://dx.doi.org/10.6084/m9.figshare. 103765

Total ascorbic acid of Col-0 WT, vtc1-1, and D3 suppressor mutants $(\mu \mathrm{mol} \mathrm{gFW}-1)$

1 Data File

http://dx.doi.org/10.6084/m9.figshare.103766

svt2 has similarities with the Ler phenotype, but has also phenotypic characteristics that are distinct from Ler The first observation we made when characterizing svt $2 \mathrm{M}_{1}$ plants was that svt2 exhibited a phenotype reminiscent of the Ler ecotype with the characteristic round leaves and erect morphology when compared to Col (Figure 3A). However, svt2 also had features that were distinct from the Ler phenotype, including overall enlarged vegetative and reproductive morphology (insets of rosettes and flowers in Figure 3A). In addition, $s v t 2$ was strongly delayed in flowering compared to the Col-0 and Ler-0 wild types and the vtcl-1 mutant (Figure 3A, 3B). Primary inflorescences in four-week-old plants were 1.4-times significantly longer in the $v t c 1-1$ mutant and approximately twice as
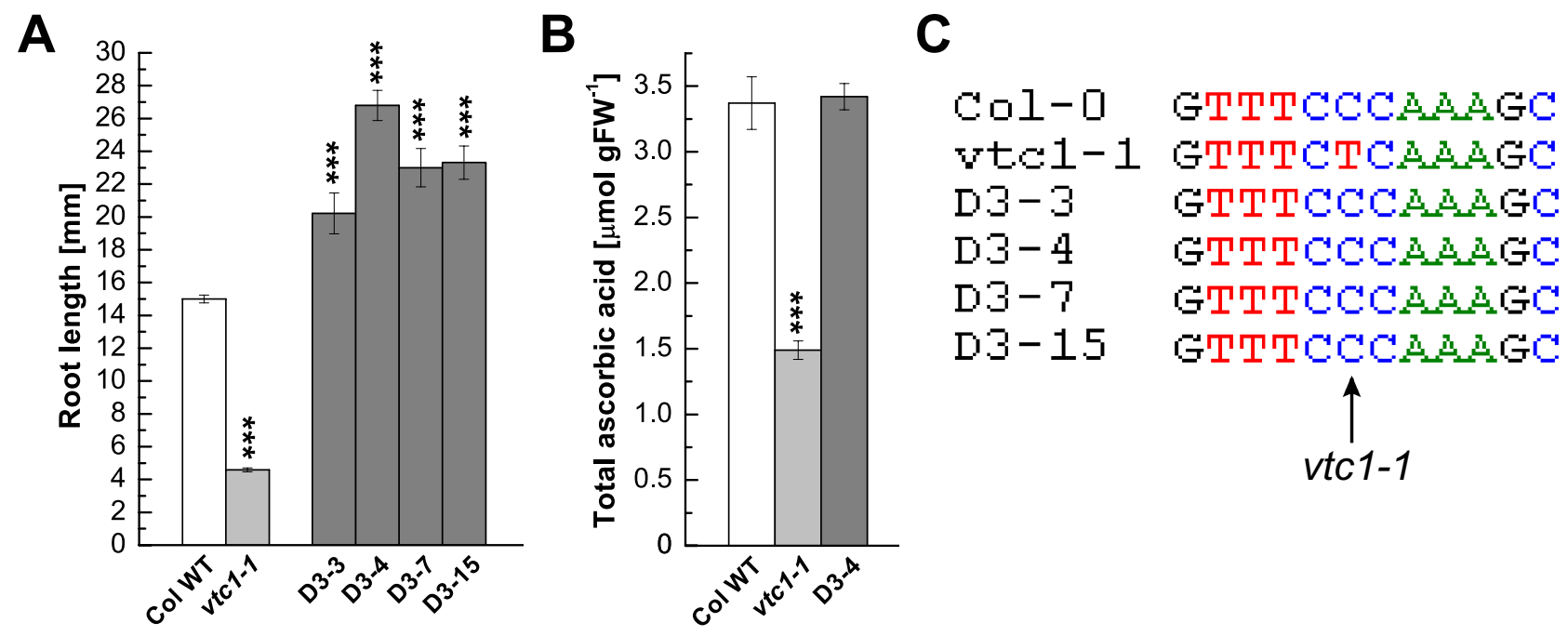

Figure 2. Phenotypic and genotypic characterization of additional vtc1-1 suppressor mutants. (A) Root length in seven-day-old seedlings grown on $1 \times$ MS. Bars represent means \pm SE of 18-73 individuals. Since D3-4 homogenously produced long roots, all individuals were included in the calculations. As D3-3, D3-7, and D3-15 developed long and short roots in an approximate 3:1 ratio, only individual seedlings that produced long roots were included in the calculations. (B) Total ascorbic acid content per gram fresh weight in whole rosettes of three-week-old plants. Bars represent means \pm SE of three (Col-0 and vtc1-1) or 24 individual replicates. ${ }^{* * *} P<0.001$ by Student's $t$-test indicates significant differences in comparison to the Col-0 wild type. (C) Sequences of the Col-0 wild type, the vtc1-1 mutant and four suppressor mutants. The arrow points to the vtc1-1 mutation, a conversion of cytosine to a thymine. 
long in the Ler-0 wild type compared to the Col-0 wild type. In svt 2 mutant plants, however, buds of primary inflorescences only began to emerge when plants were four weeks old (Figure 3A, 3B). The flowering data are consistent with previous reports, with Ler-0 wild type entering the reproductive phase before Col- 0 wild type. An early flowering phenotype of $v t c 1-1$ has been reported previously ${ }^{3}$.

The AA content in $s v t 2$ was similar to levels quantified in Col-0 and Ler-0 wild types, whereas vtcl-1 contained approximately $30 \%$ of the AA content as expected ${ }^{14,15}$ (Figure 3C). Finally, we investigated whether svt 2 also exhibits recovered root development in the presence of ammonium by growing the four genotypes in full strength $1 \times$ MS medium. The $v t c 1-1$ mutant is conditionally hypersensitive to ammonium ${ }^{8,9,11}$. Figure 3D illustrates that root length in $s v t 2$ was the same as in Col-0 wild type, whereas root development was strongly inhibited in $v t c 1-1$ as expected.

The enlarged morphology of svt 2 raises the question as to whether $s v t 2$ is polyploid. In order to test this, we assessed the size of pollen grains from the Col-0 and Ler-0 wild-types and $v t c 1-1$ and $s v t 2$ mutants. As is shown in Figure 4, pollen grains of the four genotypes are similar in size. In addition, using qPCR, we determined the number of $V T C 1$ gene copies in the four genotypes. Our results revealed that VTC1 is present as a single copy gene in both the Col-0 and Ler- 0 wild types and in the vtc1-1 and svt2 mutants (Table 2). Although an extensive chromosome analysis has not yet been performed in $s v t 2$, our results suggest that the mutant does not contain additional sets of chromosomes.

Taken together, based on the phenotypic observations, our data suggest that $s v t 2$ represents a novel $v t c 1-1$ suppressor mutant with recovered AA content and root development. Next, we characterized $s v t 2$ genotypically in order to determine whether $s v t 2$ represents an intragenic or extragenic suppressor.

\section{svt2 shares genome similarity with Ler}

To determine whether svt 2 represents an intragenic suppressor, i.e., to test whether the suppressor mutation is present within the VTC1 gene, we designed nine overlapping PCR primer sets spanning the entire $V T C 1$ gene and approximately $500 \mathrm{bp}$ of the promoter region directly upstream of the first base in the 5' UTR (Table 1, Figure 5A). PCR products were generated from genomic DNA extracted from Col-0 and Ler- 0 wild types, and $v t c 1-1$ and $s v t 2$ mutants. In eight of the nine primer pairs covering the entire VTC1 gene, the PCR products generated using svt2 genomic DNA had the same electrophoretic mobility as those generated using Col-0 wild-type genomic DNA. However, this was not the case for the first primer set. The G1F/ G1R primer set, used to amplify the VTC1 promoter region, generated a larger PCR product in $s v t 2$ than in the wild type (Figure 5B, Figure 6). The PCR product in the wild type was $567 \mathrm{bp}$, whereas that in $s v t 2$ had a size of approximately $850 \mathrm{bp}$, suggesting that $s v t 2$ contained an approximately $300 \mathrm{bp}$ insertion in this region. We repeated the PCR analysis of the VTC1 promoter region using the G1F-G1R and the G1F-G2R primer sets that should generate a PCR product of $567 \mathrm{bp}$ and $751 \mathrm{bp}$, respectively (Figure 5A). The expected size was obtained for the Col- 0 wild type and the vtc1-1 mutant. However, approximately $300 \mathrm{bp}$ larger PCR products were detected in the $s v t 2$ mutant and the Ler- 0 wild type (Figure 5B), suggesting a Ler insertion polymorphism. Thus, these data imply that svt 2 shares both phenotypic and genotypic similarities with Ler.

We therefore assessed five additional Insertion/Deletion (InDel) polymorphisms randomly chosen across the five Arabidopsis chromosomes (Table 1) in svt 2 compared to the Col-0 and Ler-0 wild types and sequenced the entire VTCl gene and the promoter region tested. Our data show that the PCR products generated for those five InDels using svt2 genomic DNA had the same electrophoretic mobility as those produced from Ler-0 genomic DNA (Figure 7). Moreover, sequence analysis of the VTC1 gene and promoter region revealed that $s v t 2$ contained a 283 bp insertion in the VTC1 promoter (Figure 5C). The insertion is highlighted in gray in Figure S1. Note additional single nucleotide polymorphisms as indicated by upright arrows in Figure 5C and Figure S1. When we aligned the VTC1 gene sequence obtained from $s v t 2$ with that of the $v t c 1-1$ mutant, the VTC1 Col-0 gene sequence deposited in the TAIR database, and the $V T C 1$ Ler GenBank sequence, the VTC1 gene sequence in svt 2 shared similarities with Ler (upright arrows in Figure 5C, Figure S1) and Col (arrows pointing down in Figure S1). However, note that there are sequences that are unique to svt 2 and are not shared between Col, vtc1-1 or Ler (arrowheads in Figure S1). Finally, note the overlap in sequences between Col, vtcl-1, svt2 and Ler on the 5' end of the sequence flanking the insertion (at approximately base pair 1990); see left-facing horizontal black arrow in Figure S1 compared to the sequence flanking the $3^{\prime}$ end of the DNA sequence insertion (starting at base pair 2273); see right-facing horizontal black arrow in Figure S1.

Finally, most intragenic suppressor mutants still contain the original mutation in addition to the suppressor mutation. Therefore, we expected that the vtcl-1 mutation is still present in $s v t 2$. However, our sequencing analysis demonstrated that $s v t 2$ did not contain the $v t c 1-1$ mutation anymore and that the mutation reverted back to the homozygous wild-type allele (Figure 5D; green shading in Figure S1).

In summary, our data demonstrate that svt2 shares DNA sequence similarity with Col and Ler, but also contains DNA sequences that are unique to this mutant. This is particularly remarkable because $s v t 2$ was generated in the $v t c 1-1$ Col- 0 background. Also, svt 2 did not contain the original vtcl-1 mutation anymore. Although our data already argue against $s v t 2$ being a result of an artifact of the experiment or a contamination with Ler, we analyzed subsequent $s v t 2$ generations and discovered additional characteristics that are unique to $s v t 2$.

Quantitative PCR to verify that VTC1 is a single copy gene in Col-0 and Ler-0 wild types and vtc1-1 and svt2 mutants

2 Data Files

http://dx.doi.org/10.6084/m9.figshare.103771

svt2 exhibits phenotypic and genotypic instability

Our initial observations revealed that approximately $10 \%$ of svt 2 $\mathrm{M}_{2}$ plants displayed a Col-like phenotype. Therefore, we planted svt $2 \mathrm{M}_{1}, \mathrm{M}_{2}$, and $\mathrm{M}_{3}$ progeny to check whether this result could be repeated and to determine segregation ratios (Table 3). Additionally, 


\section{A}

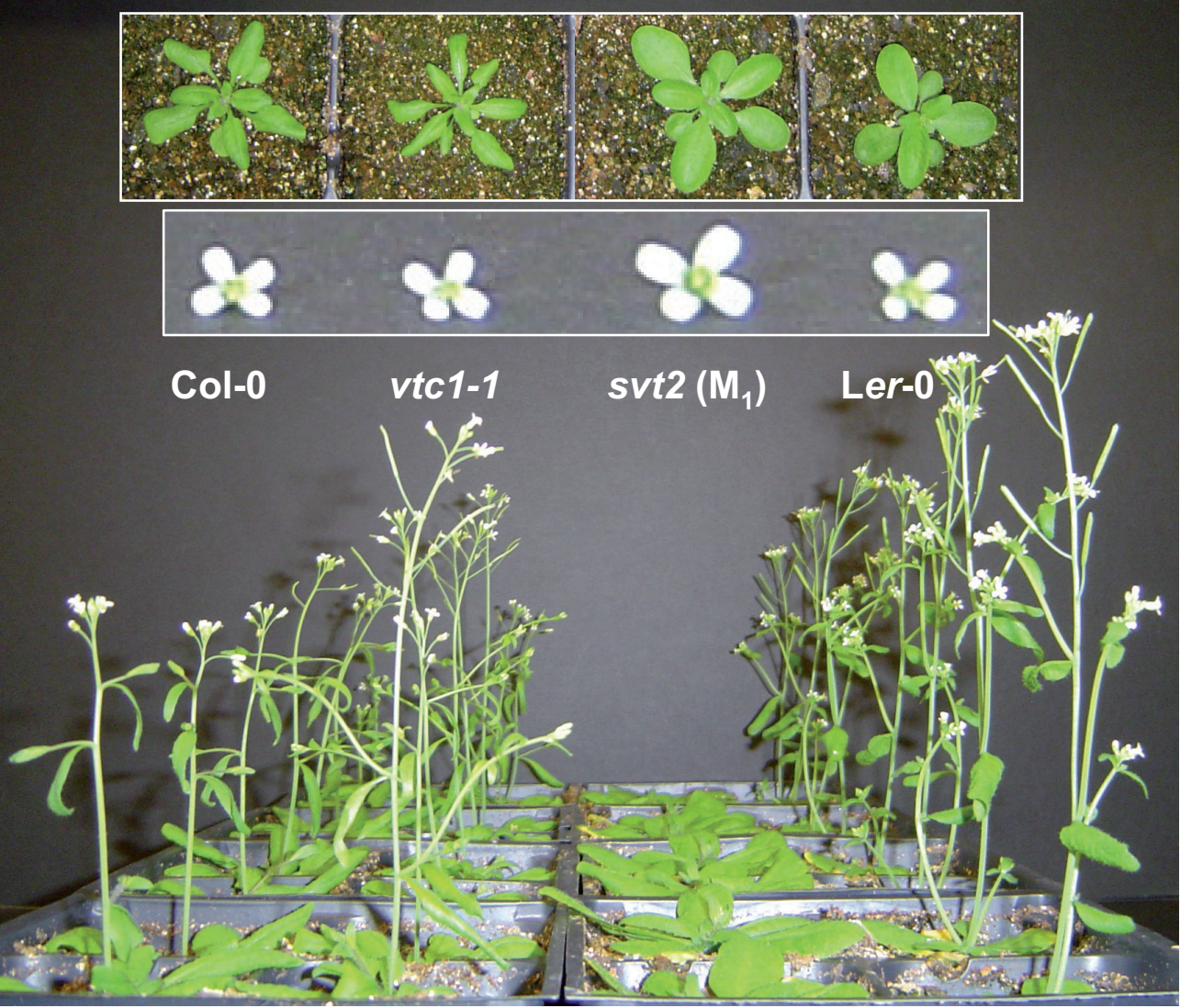

B

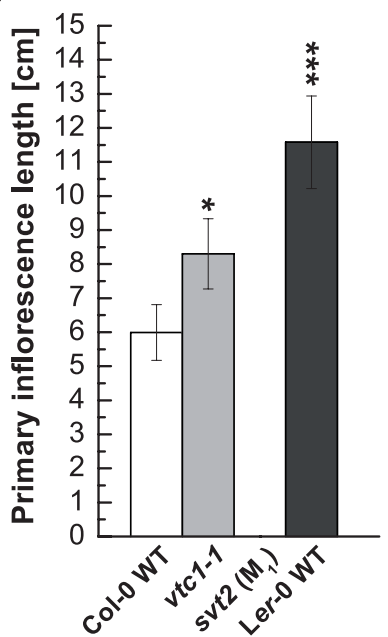

C

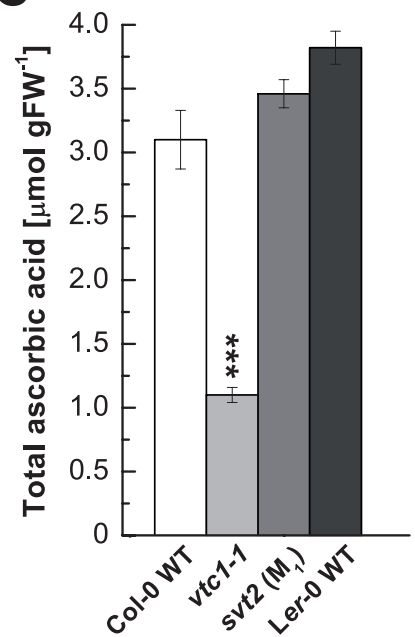

D

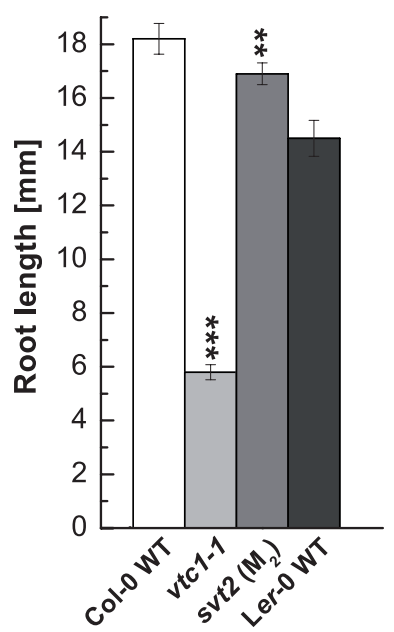

Figure 3. Phenotypic characterization of svt2. (A) Flowering phenotype of four-week-old Col-0 wild type, the vtc1-1 and svt2 mutants and the Ler-0 wild type. Insets show rosette phenotypes of the four genotypes when plants were three weeks old and the flower phenotype of six-week-old plants, respectively. (B) Primary inflorescence length when plants were four weeks old. Bars represent means \pm SE of eight individual replicates. (C) Total ascorbic acid content per gram fresh weight in whole rosettes of three-week-old plants. Bars represent means \pm SE of three individual replicates. (D) Root length in seven-day-old seedlings grown on $1 \times \mathrm{MS}$. Bars represent means $\pm \mathrm{SE}$ of $30-90$ individuals. ${ }^{\star} P<0.05,{ }^{* *} P<0.01,{ }^{* \star} P<0.001$ by Student's $t$-test indicate significant differences in comparison to Col-0 and Ler-0 wild type, respectively. 
Primary inflorescence length of Col-0 WT, vtc1-1, svt2 (M1) and Ler-0 WT (cm)

1 Data File

http://dx.doi.org/10.6084/m9.figshare.103767

Total ascorbic acid of Col-0 WT, vtc1-1, svt2 (M1) and Ler-0 WT ( $\mu \mathrm{mol} \mathrm{gFW}-1$ )

3 Data Files

http://dx.doi.org/10.6084/m9.figshare.103768

Root lengths of Col-0 WT, vtc1-1, svt2 (M2) and Ler-0 WT (mm)

3 Data Files

http://dx.doi.org/10.6084/m9.figshare.103769
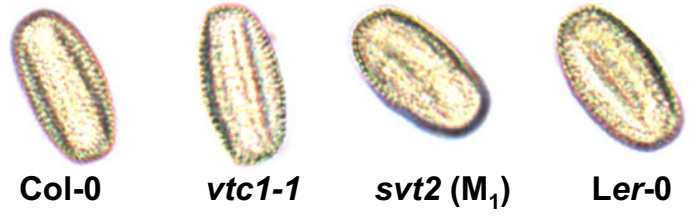

$\underline{10 \mu \mathrm{m}}$

Figure 4. Pollen phenotype of Col-0 wild type, the vtc1-1 and svt2 mutants and the Ler-0 wild type. Light images were taken when plants were 4.5 weeks old. Scale bar represents $10 \mu \mathrm{m}$.

Table 2. Quantitative PCR to verify that VTC1 is a single copy gene in Col-0 and Ler-0 wild types and vtc1-1 and svt2 mutants. Quantitative PCR was performed as described in Materials and Methods. The PAD4 gene is a known single copy gene. Therefore, an $R Q / R Q$ ratio of approximately 1 indicates that VTC 1 is present in similar quantity as PAD4, and therefore a single-copy gene.

\begin{tabular}{|c|c|c|}
\hline & $R Q / R Q$ & \\
\hline & VTC1/PAD4 & \\
\hline Col-0 WT & 0.3796 & \multirow{4}{*}{$\begin{array}{l}\text { VTC1 } \\
E=1.8\end{array}$} \\
\hline vtc1-1 & 0.5843 & \\
\hline svt2 & 0.5504 & \\
\hline Ler-0 WT & 0.6329 & \\
\hline Col-0 & 0.3153 & \multirow{4}{*}{$\begin{array}{l}\text { VTC1 } \\
\mathrm{E}=2\end{array}$} \\
\hline vtc1-1 & 0.5292 & \\
\hline svt2 & 0.4946 & \\
\hline Ler-0 WT & 0.5807 & \\
\hline
\end{tabular}

we investigated whether svt 2 progeny that were phenotypically Col-like revertants would produce svt2 (Ler-like) offspring in the next generation.

As summarized in Table 3, revertants could only be detected when a relatively large population was planted. In the $s v t 2 \mathrm{M}_{1}$ generation, only $1 \%$ of Col-like revertants were detected. In contrast, $8-10 \%$ of svt $2 \mathrm{M}_{2}$ plants displayed a Col-like phenotype, whereas no revertants were detected in the $s v t 2 \mathrm{M}_{3}$ generation. These Col-like revertants were isolated and seeds were collected from individual plants and the phenotype of the progeny in the $\mathrm{M}_{3}$ generation was assessed in some examples. In most cases, reversion appeared to be stable, i.e., once $s v t 2$ plants reverted, displaying a Col-like phenotype in the $\mathrm{M}_{2}$ generation, their $\mathrm{M}_{3}$ progeny continued to appear as Col-like plants. This was the case for the $M_{3}$ progeny of the A8 and G7 plants listed in Table 3. However, out of 63 progeny from the $\mathrm{K} 1$ revertant plant, one reverted back to a svt2-like phenotype (Table 3), i.e., the K1 double revertant switched from svt2 phenotype in the $\mathrm{M}_{1}$ generation to a Col-like phenotype in the $\mathrm{M}_{2}$ generation, and then reverted back to a svt2-like phenotype in the $\mathrm{M}_{3}$ generation. Note that only a small number of progeny was planted. In a second experiment, the $s v t 2$ Col R1 revertant produced 20 individuals displaying a svt2-like phenotype (Table 3 ). This represents a larger reversion percentage than in the $\mathrm{K} 1$ double revertant $(22.7 \%$ vs. $1.6 \%)$. This may be explained by the genotypic make-up of the Col-like reverted parents and will be presented in the next section. Figure 8 illustrates the phenotypic appearance of three examples of $s v t 2 \rightarrow$ Col single revertants (Col R1, $\mathrm{Col} \mathrm{R} 2, \mathrm{~K} 1 \mathrm{Col} \mathrm{R})$ and a svt $2 \rightarrow \mathrm{Col} \rightarrow$ svt2 double revertant $(\mathrm{K} 1$ Col R svt2 R).

Next we tested whether a Col-like revertant phenotype correlated with a Col-like genotype. Likewise, we would expect that a $s v t 2 \rightarrow$ $\mathrm{Col} \rightarrow s v t 2$ double revertant phenotype corresponds with $s v t 2$-like genomic markers. To check this we isolated genomic DNA from Col-0 and Ler-0 wild types, svt2, vtc $1-1$ and revertant mutants, and PCR-amplified the five randomly selected InDel polymorphisms plus the InDel polymorphism in the VTC1 promoter (Table 1). In all cases but the $s v t 2 \mathrm{M}_{2} \mathrm{Col} \mathrm{R} 1$ revertant, the svt2-like revertant plants (labeled svt $2 \mathrm{M}_{2}$ Col revertants 1 through 5) produced PCR products that where of the same electrophoretic mobility as the PCR products generated using Col-0 wild-type genomic DNA. In contrast, svt $2 \mathrm{M}_{1}$ plants and $s v t 2 \mathrm{M}_{2}$ plants that displayed an $s v t 2$ phenotype, gave rise to PCR products that were of the same electrophoretic mobility as those of the Ler wild type (Table 4, Figure 9). In addition, the double revertant plant K1 (labeled svt2 $M_{2} \mathrm{~K} 1 \mathrm{Col} R$ ) was genotyped in both its $\mathrm{M}_{2}$ and $\mathrm{M}_{3}$ generations. The K1 plant produced InDel PCR products similar to those of the Col- 0 wild type in the $M_{2}$ generation. However, the $M_{3}$ generation that displayed svt2-like morphology produced PCR products that were comparable to the InDel PCR products generated using Ler genomic DNA (Table 4). The svt2 $\mathrm{M}_{2}$ Col R1 (highlighted in red in Table 4 is intriguing, because it appears to contain DNA that is similar to both $\mathrm{Col}$ and Ler genomic DNA. This suggests the presence of chimeric genome sectors, which may explain the higher percentage of Col-like revertants compared to svt $2 \mathrm{M}_{2} \mathrm{~K} 1$ 


\section{A}
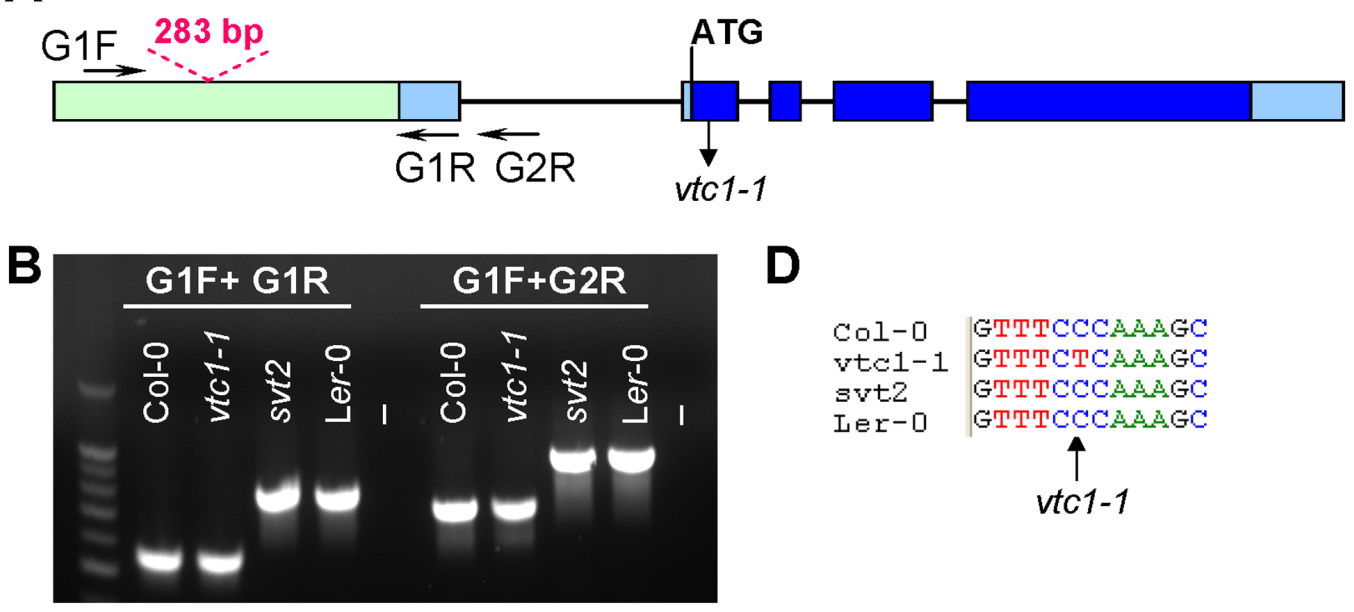

D

Q

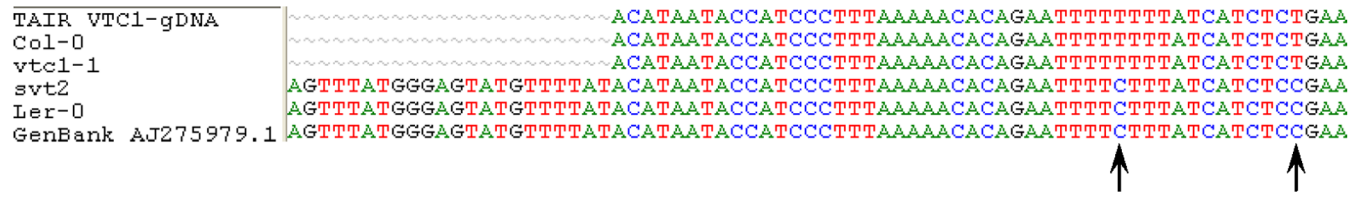

Figure 5. Genotypic characterization of sut2. (A) VTC1 Col-0 gene model. Light green box indicates VTC1 gene promoter region, light blue rectangles indicate $5^{\prime}$ and $3^{\prime}$ UTRs, dark blue rectangles indicate exons, and lines indicate introns. Shown is the location of the vtc $1-1$ mutation within the first exon, primer locations, and polymorphism insertion of 283 bp in Ler-0 VTC1. (B) PCR amplification of the VTC1 promoter region in the Col-0 wild type, vtc1-1 and svt2 mutants and Ler-0 wild type. (-) indicates negative control, no DNA. (C) Partial sequence alignment of the VTC1 promoter region from the TAIR database (Col-0), sequenced Col-0 wild type, vtc1-1 and svt2 mutants, sequenced Ler-0 wild type and the Ler-0 sequence obtained from GenBank. The alignment shows the sequence insertion in the svt2 mutant, the Ler-0 wild type and the GenBank sequence. Arrows indicate single nucleotide polymorphisms between the Ler-0 and Col-0 sequence. (D) Point mutation in vtc1-1, a conversion from a cytosine to a thymine.

PCR amplification of the VTC1 promoter region in the Col-0 wild type, vtc1-1 and svt2 mutants

1 Data File

http://dx.doi.org/10.6084/m9.figshare.103770

Col-0
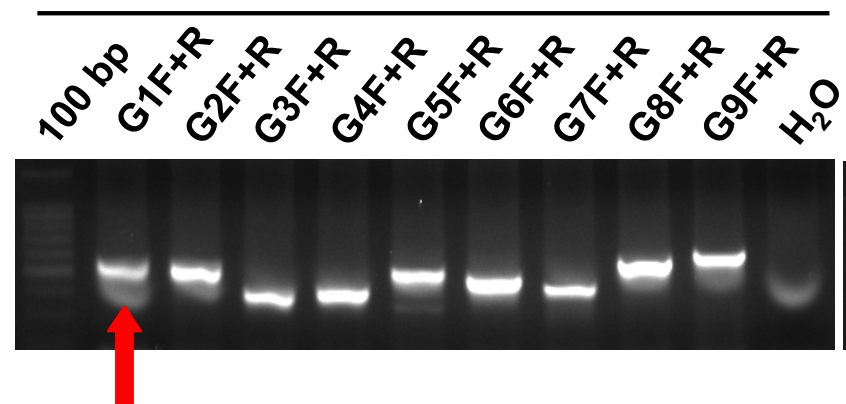

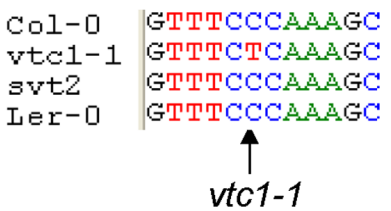

vtc1-1

Figure 6. Molecular characterization of svt2. Amplification of the VTC1 gene including $~ 500$ bp of the promoter region using a series of nine, overlapping primers ( $\mathrm{G} 1 \mathrm{~F}+\mathrm{R}$ through $\mathrm{G} 9 \mathrm{~F}+\mathrm{R})$ in both Col-0 wild type and svt2 $\mathrm{M}_{1}$ mutant genomic DNA. The last lane in each gel contained a negative control (water instead of DNA). Red arrows indicate the different sized PCR products using the same primer set. 


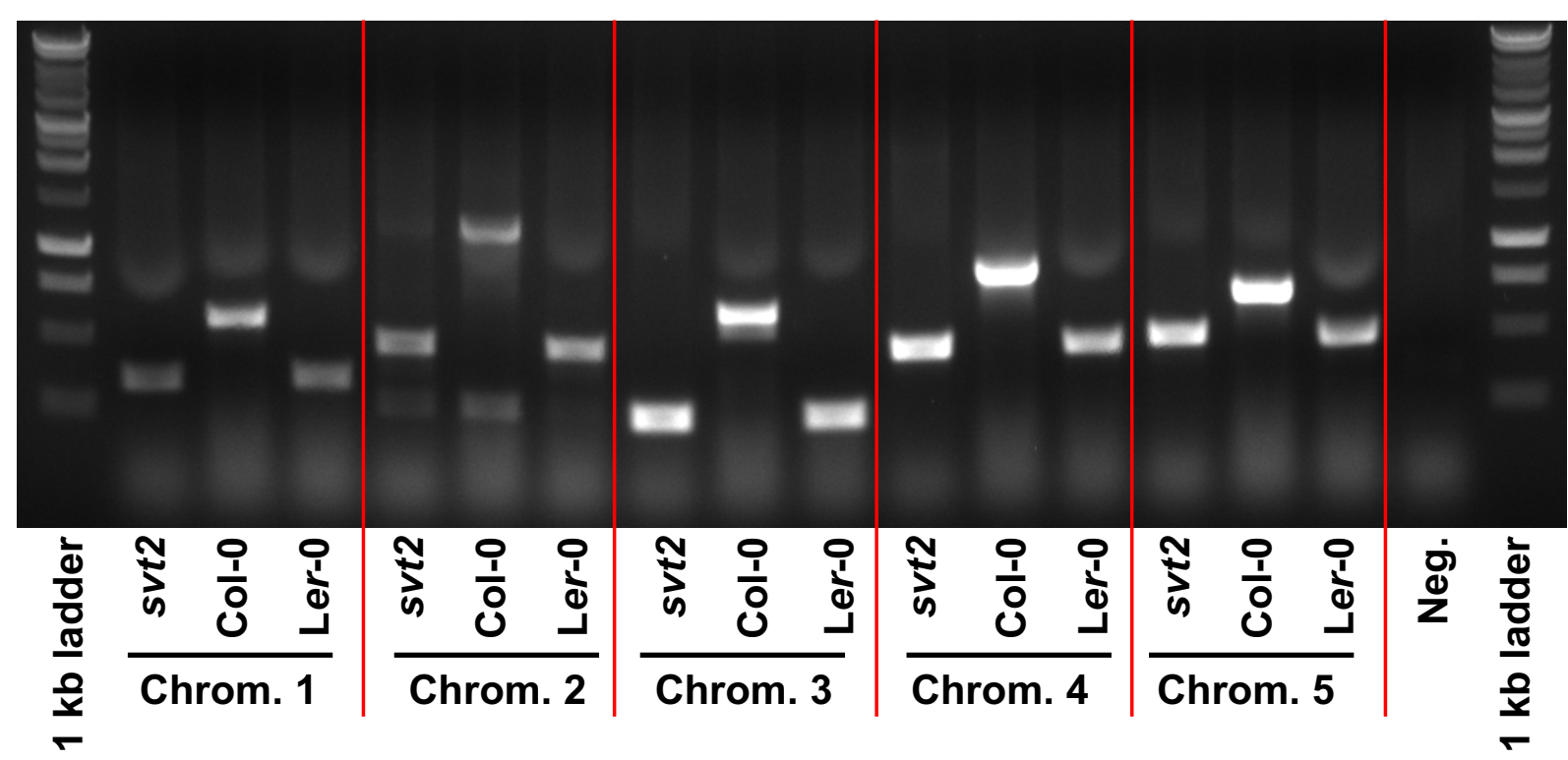

Figure 7. Insertion/Deletion polymorphism analysis in svt2, Col-0 and Ler-0. Primers were designed for five randomly selected InDel polymorphisms across the five Arabidopsis chromosomes. The polymorphisms represent insertions in Col-0 and deletions in Ler.

Table 3. Summary of revertant data. The table summarizes the number of plants screened in each of three svt2 generations $\left(M_{1}, M_{2}\right.$ and $\left.M_{3}\right)$, screens of revertant progeny from Col-like revertants $(A 8, G 7, K 1)$, and the revertant progeny of a Ler-like line ( $\mathrm{K} 1 \mathrm{Col} \mathrm{R}$ svt2 R). The percent reversion is shown in the last column. Although the number of progeny plants tested is relatively large, some lines did not give rise to revertant progeny. $\mathrm{R}$ denotes revertant. *indicates mutant plants that were also analyzed genotypically (see Table 4)

\begin{tabular}{|c|c|c|c|c|}
\hline Experiment & Generation & Total \# of plants & \# of phenotypic revertants & $\%$ reversion \\
\hline \multirow{3}{*}{1} & svt2 $\mathrm{M}_{1}$ & 63 & 0 & 0 \\
\hline & $\begin{array}{l}\text { svt2 } \mathrm{M}_{2}, 3 \text { of } 7 \text { revertants tested further: } \\
\text { st2 } \mathrm{A} 8 \mathrm{Col} \mathrm{R} \mathrm{M}_{3} \\
\text { svt2 } \mathrm{G} 7 \mathrm{Col} \mathrm{R} \mathrm{M}_{3} \\
\text { svt2 } \mathrm{K} 1 \mathrm{Col} \mathrm{R} \mathrm{M}_{3}{ }^{\star} \\
\text { svt2 } \mathrm{K} 1 \mathrm{Col} R \text { svt2 } \mathrm{R} \mathrm{M}_{4}{ }^{*}\end{array}$ & $\begin{array}{l}78 \\
64 \\
64 \\
63 \\
96\end{array}$ & $\begin{array}{l}7 \text { (Col phenotype) } \\
0 \\
0 \\
1 \text { (svt2 phenotype) } \\
0\end{array}$ & $\begin{array}{l}8.97 \\
0 \\
0 \\
1.58 \\
0\end{array}$ \\
\hline & svt2 $\mathrm{M}_{3}$ & 96 & 0 & 0 \\
\hline & svt2 $\mathrm{M}_{1}$ & 96 & 1 (Col phenotype) & 1.04 \\
\hline 2 & $\begin{array}{l}\text { svt2 } \mathrm{M}_{2}, 2 \text { of } 5 \text { revertants tested further: } \\
\text { svt2 } \mathrm{Col} \mathrm{R} 1 \mathrm{M}_{3}{ }^{*} \\
\text { svt2 Col R4 } \mathrm{M}_{3}^{*}\end{array}$ & $\begin{array}{l}62 \\
88 \\
96\end{array}$ & $\begin{array}{l}5 \text { (Col phenotype) } \\
20 \text { (svt2 phenotype) } \\
0\end{array}$ & $\begin{array}{l}8.06 \\
22.73 \\
0\end{array}$ \\
\hline 3 & svt2 $\mathrm{M}_{2}$ & 96 & 10 (Col phenotype) & 10.42 \\
\hline
\end{tabular}

Col R. Note that the PCR results are in line with the sequencing analysis of the revertants. That is, Col-like revertants and svt2-like revertants share sequence similarity with Col-0 and Ler wild type, respectively (Figure S2).

Taken together, these data suggest (i) transgenerational phenotypic and genotypic instability in $s v t 2$, and that (ii) $s v t 2$ offspring do not segregate in a Mendelian fashion. In an attempt to obtain first insights toward a mechanism that is causing this genotypic instability, we investigated whether transgenerational epigenetic inheritance could play a role.
Genome instability in svt2 does not appear to be triggered by a transgenerational epigenetic mechanism

To investigate whether genome instability is caused by transgenerational epigenetic inheritance in the svt 2 mutant, we performed reciprocal crosses between $s v t 2$ mutants and Col-0 wild-type plants. It is possible that through the EMS mutagenesis of vtc1-1 seeds, genes involved in the regulation of epigenetic alterations were altered, whereby their activity was affected. There is increasing evidence in both plants and animals that epigenetic marks are not always cleared between generations. Incomplete erasure at genes associated with a measurable phenotype results in unusual patterns of inheritance 


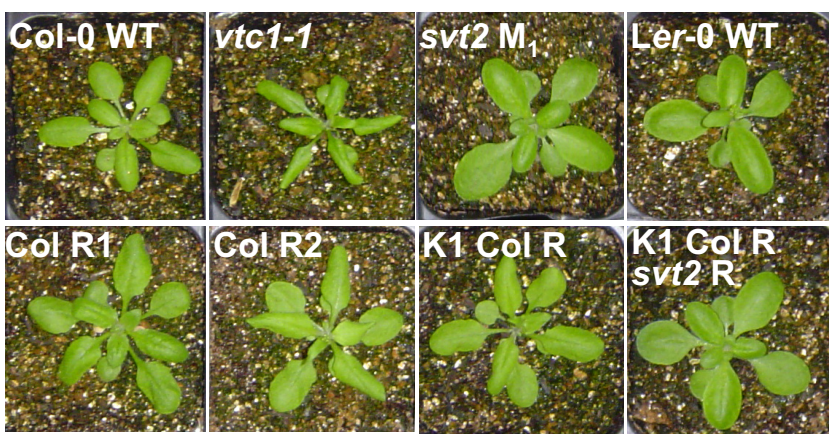

Figure 8. Phenotype of svt2 revertants. Plants were three weeks old when photographs were taken. Top row represents controls, Col-0 wild type, vtc $1-1$ and svt2 mutants, and Ler-0 wild type. Bottom row represents three Col-like revertants, svt2 Col R1 $\mathrm{M}_{3}$, svt2 Col R2 $\mathrm{M}_{3}$, svt2 $\mathrm{K} 1 \mathrm{Col} \mathrm{R}_{3}$, and a double revertant, svt2 $\mathrm{K} 1 \mathrm{Col} \mathrm{R}$ svt2 $\mathrm{R} \mathrm{M}_{4}$. $\mathrm{R}$ stands for revertant.

Table 4. Summary of PCR-based molecular genotypes. With the exception of svt2 Col R1 $\mathrm{M}_{2}$, where Col and Ler markers and one heterozygous marker were found (highlighted in red), phenotype matched genotype. That is, a Col-like phenotype correlated with the presence of Col polymorphisms, while a Ler-like phenotype correlated with Ler polymorphisms. C, L, and $\mathrm{H}$ refer to Col, Ler, or heterozygous, respectively. $\mathrm{R}$ denotes revertant. n.d., not detected.

\begin{tabular}{|c|c|c|c|c|c|c|}
\hline Genotype & $\begin{array}{l}\text { InDel } 1 \\
450919\end{array}$ & $\begin{array}{l}\text { InDel } 2 \\
451470\end{array}$ & $\begin{array}{l}\text { InDel } 3 \\
469762\end{array}$ & $\begin{array}{l}\text { InDel } 4 \\
449053\end{array}$ & $\begin{array}{l}\text { InDel } 5 \\
455100\end{array}$ & $\begin{array}{l}\text { G1F + } \\
\text { G2R VTC1 }\end{array}$ \\
\hline Col-0 WT & C & C & C & C & C & C \\
\hline$v t c 1-1$ & C & C & C & C & C & C \\
\hline Ler-0 WT & L & L & L & L & L & L \\
\hline svt2 $\mathrm{M}_{1}$ & L & L & L & L & L & L \\
\hline svt2 $\mathrm{M}_{2}$ & L & L & L & L & L & L \\
\hline svt2 Col R1 M 2 & C & L & C & $\mathrm{H}$ & C & C \\
\hline svt2 Col R2 $\mathrm{M}_{2}$ & C & C & C & C & C & C \\
\hline svt2 Col R3 $\mathrm{M}_{2}$ & C & C & C & C & n.d. & C \\
\hline svt2 Col R4 $\mathrm{M}_{2}$ & C & C & C & C & C & C \\
\hline svt2 Col R5 $\mathrm{M}_{2}$ & C & C & C & C & C & C \\
\hline svt2 $\mathrm{K} 1 \mathrm{Col} \mathrm{R} \mathrm{M}_{2}$ & C & C & C & C & C & C \\
\hline $\begin{array}{l}\text { svt2 } \mathrm{K} 1 \mathrm{Col} \mathrm{R} \\
\text { svt2 } \mathrm{R} \mathrm{M}_{3}\end{array}$ & L & L & L & L & L & L \\
\hline
\end{tabular}

Summary of PCR-based molecular genotypes

1 Data File

http://dx.doi.org/10.6084/m9.figshare.103772

Summary of PCR-based molecular genotypes

1 Data File

http://dx.doi.org/10.6084/m9.figshare.103773 from one generation to the next, termed transgenerational epigenetic inheritance ${ }^{22,23}$. Therefore, analysis of the progeny of the reciprocal crosses is expected to provide some first insights on the possibility of transgenerational epigenetic inheritance that is transmitted maternally. If this were the case, only progeny of crosses with a maternal svt 2 donor should have a svt2-like phenotype. To determine the genotypes of the $F_{1}$ progeny of the reciprocal crosses, we performed another InDel polymorphism assay as described above. In addition, progeny were also screened using the VTCl InDel promoter polymorphism. Table 5 contains a summary of the InDel screen for progeny from each reciprocal cross. In all but six of the progeny from the reciprocal crosses, PCR products similar to those obtained using Col and Ler genomic DNA, respectively, were generated, suggesting that the $\mathrm{F}_{1}$ of the reciprocal crosses were heterozygous. A similar result was obtained for the VTC1 promoter polymorphism marker in all reciprocal crosses. Note, however, that for some polymorphisms and irrespective of whether svt 2 or Col- 0 served as female or male donor, respectively, PCR products comparable to those obtained using Ler-0 wild-type DNA were prevalent (highlighted in red in Table 5). This is surprising because heterozygosity was expected at all loci. This suggests that some parts of the genome were not inherited equally from both parents. Taken together, these results suggest that maternal epigenetic inheritance may not be the cause of genome instability in $s v t 2$. However, at some loci $s v t 2$-like alleles dominate over Col-0.

\section{Discussion}

The svt 2 mutant was initially identified as a putative suppressor of the AA-deficient Arabidopsis mutant vtcl-1, as was evident in wild-type levels of AA (Figure 3C) and recovered root development in the presence of ammonium (Figure 3D). However, svt2 manifests other interesting characteristics, including genotypic and phenotypic instability. These unique features could aid in our understanding of the complex mechanisms controlling genome instability and restoration.

svt2 is a novel Arabidopsis mutant and not a result of an experimental artifact, seed contamination, or outcrossing Several lines of evidence support our findings that $s v t 2$ is a novel mutant. First, svt 2 was the only suppressor mutant isolated among over 1000 EMS-mutagenized $\mathrm{M}_{0}$ seeds to show unique phenotypic characteristics. Astonishingly, our genetic analysis revealed that both maternal and paternal alleles were affected in five randomly selected InDel polymporphism loci, the newly discovered InDel polymporphism in the VTC1 promoter, and additional SNPs (Figure 5B-D, Figure 6, Figure S1). These data demonstrate that $s v t 2$ has acquired new characteristics, presumably as a result of EMS mutagenesis, and that $s v t 2$ is neither Col nor Ler. These data also argue against $s v t 2$ being an experimental or PCR artifact.

Second, a number of data provide strong arguments against seed contamination. (1) With high reproducibility, descendents of the original $s v t 2$ mutant produce offspring revertants with Col-like features (Table 3, Table 4; Figure 8, Figure 9). (2) One of the Col-like revertants, svt $2 \mathrm{Col} \mathrm{R} 1 \mathrm{M}_{3}$, exhibited heterozygosity at some of the InDels tested (Table 4). (3) One of those Col-like revertants, svt $2 \mathrm{~K} 1 \mathrm{Col} \mathrm{R} \mathrm{M}$, produced progeny that reverted 


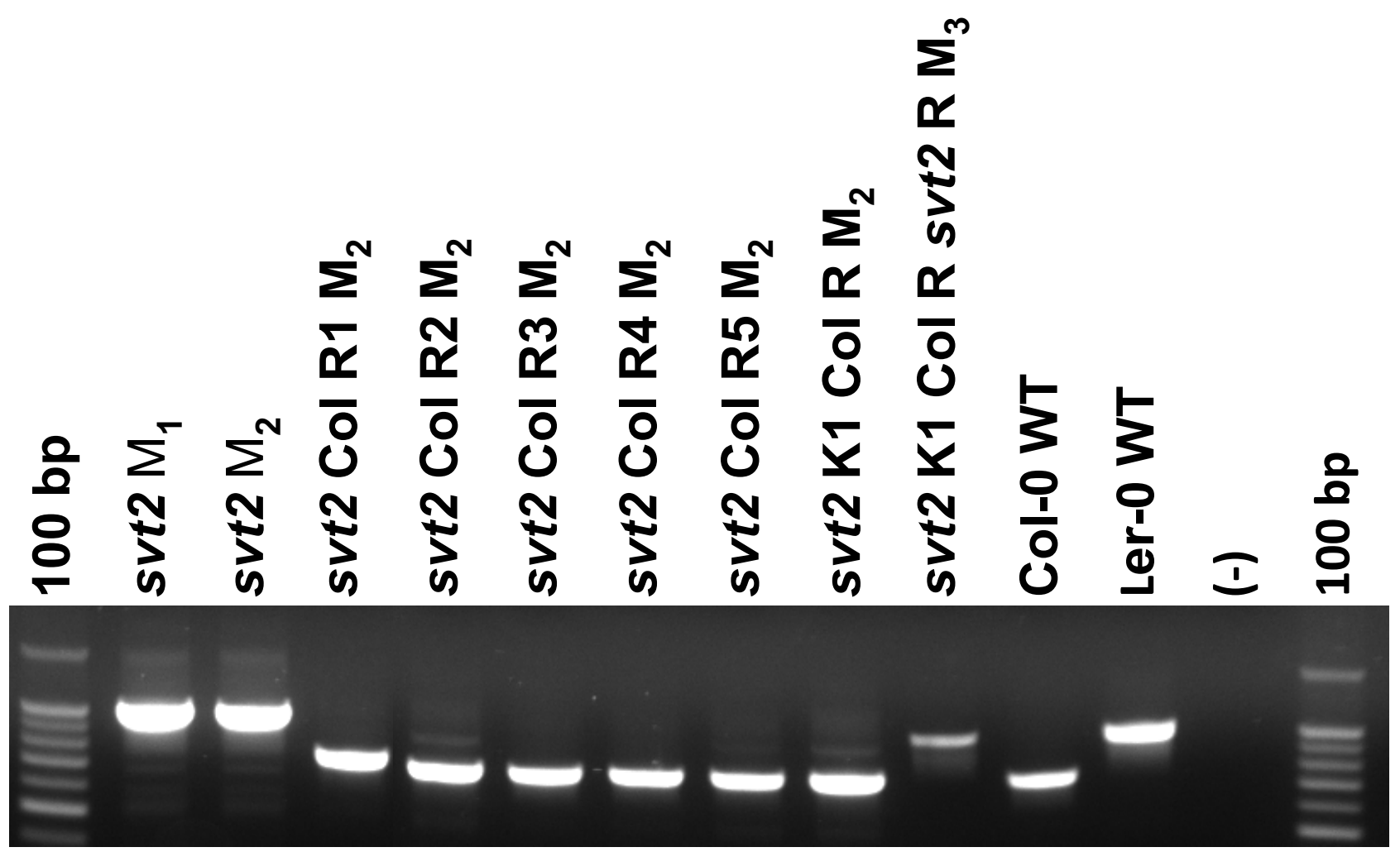

Figure 9. Insertion/Deletion polymorphism analysis in svt2, Col-0, Ler-0, and revertants. PCR amplification of the Col/Ler VTC1 promoter polymorphism in svt2 plants and svt2 revertant (R) plants, amplified with the VTC1 G1F and G2R primers. (-) indicates negative control, no DNA.

Table 5. Reciprocal crosses between svt2 and Col-0 wild-type lines. Molecular analysis of the InDel polymorphism markers showed evidence of cryptic but persistent homozygosity, irrespective of the direction of the sexual cross (highlighted in red). However, heterozygosity was expected at all loci.

Female $\times$ Male $\quad$ InDel $1 \quad$ InDel $2 \quad$ InDel $3 \quad$ InDel $4 \quad$ InDel $5 \quad$ G1F + G2R crosses $\quad 450919451470469762449053455100$ VTC1

\begin{tabular}{|c|c|c|c|c|c|}
\hline svt2 $\times$ Col- $0 \mathrm{~F}_{1} 1 \mathrm{H}$ & $\mathrm{H}$ & $L$ & $\mathrm{H}$ & $\mathrm{H}$ & $\mathrm{H}$ \\
\hline svt $2 \times \mathrm{Col}-0 \mathrm{~F}_{1} 2 \mathrm{H}$ & $\mathrm{H}$ & $L$ & $\mathrm{H}$ & $\mathrm{H}$ & $\mathrm{H}$ \\
\hline svt $2 \times \mathrm{Col}-0 \mathrm{~F}_{1} 3 \mathrm{H}$ & $\mathrm{H}$ & $\mathrm{H}$ & $\mathrm{H}$ & $\mathrm{H}$ & $\mathrm{H}$ \\
\hline svt $2 \times \mathrm{Col}-\mathrm{OF}_{1} 4 \mathrm{H}$ & $\mathrm{H}$ & $\mathrm{H}$ & $\mathrm{H}$ & $\mathrm{H}$ & $\mathrm{H}$ \\
\hline Col- $0 \times$ svt $2 \mathrm{~F}_{1} 1 \mathrm{H}$ & $\mathrm{H}$ & $\mathrm{H}$ & $L$ & $\mathrm{H}$ & $H$ \\
\hline Col-0 $\times$ svt2 $\mathrm{F}_{1} 2 \mathrm{H}$ & $\mathrm{H}$ & $L$ & $\mathrm{H}$ & $\mathrm{H}$ & $\mathrm{H}$ \\
\hline Col-0 $\times$ svt2 $\mathrm{F}_{1} 3 \mathrm{H}$ & $\mathrm{H}$ & $\mathrm{H}$ & $L$ & $\mathrm{H}$ & $\mathrm{H}$ \\
\hline Col-O $\times$ svt2 $\mathrm{F}_{1} 4 \mathrm{H}$ & $\mathrm{H}$ & $\mathrm{L}$ & $\mathrm{H}$ & $\mathrm{H}$ & $\mathrm{H}$ \\
\hline
\end{tabular}

Reciprocal crosses between svt2 and Col-0 wild-type lines

1 Data File

http://dx.doi.org/10.6084/m9.figshare.103774
Repeated PCR reactions of reciprocal crosses between svt2 and Col-0 wild-type lines

1 Data File

http://dx.doi.org/10.6084/m9.figshare.103775

Reciprocal crosses between svt2 and Col-0 wild-type lines

1 Data File

http://dx.doi.org/10.6084/m9.figshare.103776

Repeated reciprocal crosses between svt2 and Col-0 wild-type lines

1 Data File

http://dx.doi.org/10.6084/m9.figshare.103777

back to svt2-like plants (Table 3, Table 4; Figure 8, Figure 9). (4) We were unable to obtain true $F_{1}$ heterozygotes in all svt2/ Col-0 reciprocal crosses (Table 5). (5) Delayed flowering and enlarged morphology phenotypes argue against the fact that svt2 is a result of a Ler-0 wild-type seed landing on the flat during the 
initial planting of the $v t c 1-1 \mathrm{M}_{0}$ mutagenized population. There is the possibility of a Ler seed contamination of the $v t c l-1$ seed stock used for EMS mutagenesis. Although we have sequenced the $v t c 1-1$ seed stock used for this experiment and confirmed that it is homozygous for the vtcl-1 mutation, one could argue that sequencing the seed stock may not be a sensitive enough method to rule out contamination with a few Ler seed. We performed many other experiments using this very same seed stock and never observed Ler-like plants among the $v t c 1$ population. However, arguments (1) through (4) above speak most compellingly against seed contamination.

Third, the following experimental evidence argues against the possibility that $s v t 2$ was generated by cross pollination of $v t c 1-1$ mutant plants with Ler wild-type plants. (1) If $s v t 2$ were generated by Ler cross-pollination, the InDel polymorphism markers tested using svt2 genomic DNA should have indicated heterozygosity. This, however, was not the case (Table 4). (2) While svt2 shares phenotypic and genotypic characteristics with Ler and Col, it also has unique features (Figure 3A, Figure S1). (3) svt2 exhibits phenotypic and genotypic instability, causing the appearance of revertants with persistent reproducibility. (4) Ler plants were not grown in our growth chambers at the time of the mutagenesis experiment. Furthermore, $s v t 2$ was isolated by placing Aracons over the mutant plant to allow self-fertilization and seed production.

\section{Possible causes of genome instability in svt2}

Our results are indicative of genome instability in svt2. Genome instability may be a result of polyploidy ${ }^{24}$. Polyploids can arise from genome duplication (autopolyploids) or interspecific hybridization (allopolyploids). Our data suggest that svt2 does not contain multiple sets of chromosomes, because VTCl occurs as a single copy gene in $s v t 2$ and vtcl-1 mutants as well as the Col-0 and Ler-0 wild-type controls (Table 2). Furthermore, extra DNA must be replicated with each cell division. Therefore, enlarged cell size is often associated with polyploids ${ }^{25}$. The chemical mutagenesis of $v t c 1-1$ seed could have resulted in mutations, which may have led to increased ploidy levels in one, two, or all three meristem layers, L1, L2, and L3. However, only mutations in the L2 layer, which gives rise to the reproductive organs, are inherited. Polyploidy in the L2 layer is reflected in pollen size. While svt 2 has an overall enlarged morphology (Figure 3A), its pollen size is comparable to that of the other three genotypes (Figure 4). This suggests that svt2 anthers are not polyploid. Finally, allopolyploids often display a greater degree of heterozygosity ${ }^{25}$, low fertility, and low embryonic viability ${ }^{26-28}$. This, however, is not the case in $s v t 2$. The fact that $s v t 2$ is fertile and that its enlarged morphology is heritable from one generation to the next suggests that $s v t 2$ is neither a somatic nor a gametic polyploid. Thus, it is therefore unlikely that polyploidy in $s v t 2$ contributes to genome instability. This is supported by Ruffio-Chable and co-workers, who reported that between $5 \%$ and $21 \%$ of $\mathrm{F}_{1}$ hybrids in Brassica oleracea showed aberrant leaf phenotypes, despite normal ploidy levels ${ }^{29}$.

Instead, we hypothesize that genome instability of $s v t 2$ was further aggravated by exposing the already instable genome of $v t c 1-1$ mutants to EMS. It has recently been shown that plants impaired in certain aspects of protection against reactive oxygen species have a higher incidence of spontaneous double-strand breaks ${ }^{30}$. The AA-deficient vtcl-1 mutant has a three-fold higher spontaneous homologous recombination frequency and has a higher incidence of double-strand breaks (see below). Similar results were reported for the Arabidopsis thaliana flavonoid-deficient mutants $t t 4$ and $t t 5^{30}$. One may speculate that through the high level of stress induced by EMS, a yet unknown mechanism of genome restoration was turned on. In fact, genome alterations in soybean and flax in response to environmental stress have been reported previously ${ }^{31,32}$. In the process of soybean cell culture, massive specific changes in numerous genome-wide loci were observed ${ }^{31}$. It was suggested that this genetic variation is a consequence of specific recombinational events. Similarly, in flax a single-copy 5.7 kilobase DNA fragment that was not present in the parent line appeared in genotrophs in response to particular growth conditions ${ }^{32}$.

\section{Possible mechanisms of genome restoration in svt2}

The experimental evidence described in this work raises the question as to what mechanism is responsible for the loss or reintroduction of genomic DNA sequences in the original svt2 mutant and its revertant offspring. Several mechanisms may be considered: activity of transposable elements, random mutations, unequal crossing over, gene conversion, double-strand breaks and recombination, and activity of an RNA cache.

Transposons are DNA elements capable of moving around the genome; movement is often associated with chromosome breaks and formation of unstable mutations, which revert frequently but often give rise to new phenotypes. Movement of transposable elements often occurs during meiosis and mitosis and is accelerated by genome damage ${ }^{33}$. These represent conditions that are present in svt2. However, transposons have a variety of molecular features that do not apply to svt2. Transposons exist as multiple copies in the genome. A blast search of the VTC1 promoter insertion in $s v t 2$ did not return any other hits, indicating that the DNA sequence is not present in its entirety anywhere else in the genome. Additionally, transposon termini represent inverted repeats. This, however, is not the case in svt2 (Figure S1). A short, direct repeat of genomic DNA often flanks the transposon, leaving a "footprint". Our sequencing analysis of the VTC1 promoter region in svt 2 did not reveal any footprints, suggesting that transposon activity is not responsible for the insertion or loss of novel sequences in $s v t 2$ (Figure S1).

Random mutations caused by EMS mutagenesis could have activated an unknown mechanism in $v t c 1-1$ seeds, giving rise to the phenome and genome instability in svt2. This may explain the novel SNPs we detected in $s v t 2$ that are distinct from the $v t c 1-1$ mutant and Col-0 and Ler-0 wild types (Figure S1). The disappearance of the $v t c 1-1$ mutation in $s v t 2$ (Figure 5D, Figure S1) may also be explained by the introduction of a random mutation. However, it is possible that exposure of vtc1-1 seeds to EMS could have reversed the original $v t c 1-1$ mutation to the wild-type sequence, as $v t c 1-1$ was initially isolated in an EMS screen ${ }^{15}$. Interestingly, Conklin and co-workers previously isolated two vtcl alleles, vtc1-1 and $v t c 1-2$, containing the exact same single cytosine to thymine point mutation at amino acid position 64 relative to the start codon, despite the fact that $v t c 1-1$ and $v t c 1-2$ mutants were isolated independently from different EMS-mutagenized pools ${ }^{15}$. The authors 
suggested that a limited number of mutations are tolerable in the VTC1 enzyme GDP-D-mannose pyrophosphorylase without causing embryo lethality. This is supported by the fact that several independently isolated cyt mutant alleles containing different amino acid mutations in VTCl are embryo lethal ${ }^{34}$. To date, only the $v t c 1-l^{15}$ and $h s n l$ mutations ${ }^{8}$ have been isolated and reportedly do not cause embryo lethality. This suggests some form of allelic constraint that has been reported in Arabidopsis previously ${ }^{35,36}$. Furthermore, in the EMS screen in which the svt 2 mutant was isolated, several other vtcl-1 suppressor mutants with restored root development in the presence of ammonium were identified. Sequencing analysis revealed that in all of these mutants the vtc 1-1 mutation was restored to the wild-type allele, while the suppressor mutants neither exhibited a svt2-like phenotype nor did they produce revertants in the subsequent generation (Kempinski et al., unpublished data).

Exposure to EMS or $\gamma$-radiation has been reported to induce high frequency phenotypic instability in the Arabidopsis disease resistance genes $C P R 1$ and $B A L$, which map to the RPP5 locus ${ }^{16}$. Yi and Richards reported destabilization of phenotypes in both the $b a l$ and cprl mutants in more than $10 \%$ of EMS-treated plants in the $\mathrm{M}_{1}$ generation. They also identified exceptions to simple Mendelian inheritance in the $\mathrm{M}_{2}$ generation. Phenotypic instability was also observed in bal $\times \operatorname{cprl} \mathrm{F}_{1}$ hybrids. The authors suggested that the high degree of phenotypic instability in bal and cprl mutants is due to the fact that the RPR5 locus can adopt different metastable genetic or epigenetic states, whose stability is highly susceptible to mutagenesis and pairing of different alleles. Yi and Richards later reported that the phenotypic instability of bal mutants is caused mainly by gene duplication and hypermutation of the $S N C 1$ gene $^{17}$.

As observed in the cprl and bal mutants, we hypothesize that EMS treatment has destabilized the genome of $s v t 2$ by interrupting one or more mechanisms involved in genomic inheritance. A combination of unequal crossing over, gene conversion, double-strand breaks, DNA recombination, and/or the presence of an RNA cache template may explain the loss and reappearance of DNA sequences in $s v t 2$. Genome-wide non-Medelian inheritance of extra-genomic information in Arabidopsis was reported in the hothead (hth) Arabidopsis mutant ${ }^{37}$. Self-fertilization of homozygous mutant plants resulted in approximately $10 \%$ hth revertants, which were $h t h / H T H$ heterozygous, suggesting that the HTH gene was altered in the progeny. However, the authors also detected rare homozygous revertants HTH/HTH embryos, which must have inherited one of their two wild-type $H T H$ genes from the maternal parent and could not have been a result of outcrossing. Inheritable genome-wide highfrequency gene homozygosity in early generations in rice has also been reported ${ }^{38}$. Lolle et al. postulated that these genetic restoration events are the result of a template-directed process that utilizes an ancestral RNA-sequence cache ${ }^{37}$. This hypothesis is supported by observations reported by $\mathrm{Xu}$ and co-workers ${ }^{38}$. Therefore, our genetic and phenotypic svt 2 data, in conjunction with the observed higher occurrence of double-strand breaks and spontaneous homologous recombination frequency in $v t c 1-1$, are in support of the RNA cache theory. Additional studies are needed to provide experimental support for this hypothesis.

\section{Conclusions}

We have isolated a novel Arabidopsis mutant that is capable of restoring genetic information that was not present in the chromosomal genome of its parents. We suggest that this ancestral information is present in some cryptic form that is accessible under extreme stress conditions. Genome restoration could be advantageous to plants that encounter environmental changes for which ancestral genes were better adapted. However, the mechanisms responsible for triggering and executing genome restoration remain to be determined. Double strand breaks, DNA recombination, and/or the activity of an RNA cache may be contributing factors. In the future, svt 2 may serve as a model to study nonMendelian inheritance and could provide insight into the evolution and diversification of Arabidopsis ecotypes.

\section{Abbreviations}

AA, ascorbic acid; EMS, ethyl methanesulfonate; InDel, Insertion/ Deletion; MS, Murashige and Skoog.

\section{Author contributions}

$\mathrm{CB}$ and $\mathrm{CFK}$ conceived the study and designed the experiments. CFK, SVC, CS and CB conducted the experiments and analyzed the data. $\mathrm{CB}$ and $\mathrm{CFK}$ prepared the first draft of the manuscript. All authors were involved in the revision of the draft manuscript and have agreed to the final content.

\section{Competing interests}

No relevant competing interests disclosed.

\section{Grant information}

This work was supported by a start-up grant of West Virginia University to $\mathrm{CB}$.

The funders had no role in study design, data collection and analysis, decision to publish, or preparation of the manuscript.

\section{Acknowledgements}

We would like to thank Dr. Patricia Conklin for providing vtcl-1 mutant and Col-0 wild-type seed. We also wish to thank Dr. Karen Weiler for allowing us to use her microscope and Dr. Rosana Schafer for providing a plate reader. 
Col-0 VTC1 TAIR gDNA

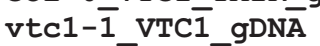
svt2_VTTC1_g $\overline{\mathrm{DNA}}$

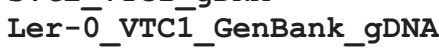

Col-0_VTC1_TAIR_gDNA

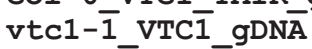
svt2 VTC1 gDNA

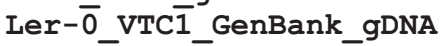

Col-0 VTC1 TAIR gDNA vtc $1-\overline{1}$ VTC $\overline{1}$ gDN $\bar{A}$ svt2 VT̄C1_gDNA

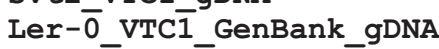

Col-0_VTC1_TAIR_gDNA

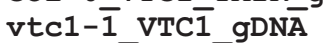
svt2 VT̄C1_gDNA

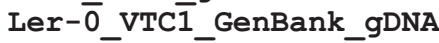

Col-0 VTC1 TAIR gDNA vtc1- $\overline{1}$ VTC $\overline{1}$ gDN $\bar{A}$ svt2 vTC1 gDNA

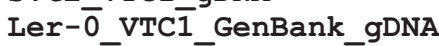

Col-0_VTC1_TAIR_gDNA vtc1- $\overline{1}$ VTC $\overline{1}$ gDN $\bar{A}$ svt2 VT̄C1 gDNA

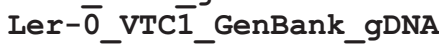

Col-0 VTC1 TAIR gDNA

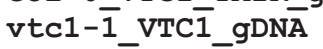
svt2 VTC1 gDNA

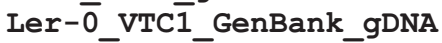

Col-0_VTC1_TAIR_gDNA

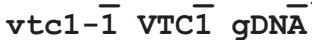
svt2 v' $\bar{T} 1$ g $\overline{D N A}$

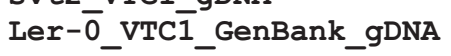

Col-0 VTC1 TAIR gDNA

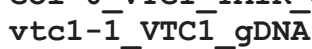
svt2 vTC1 gDNA Ler- $\overline{0}$ VTC $\overline{1}$ GenBank gDNA

$1630 \quad 1640 \quad 1650 \quad 1660 \quad 1670 \quad 1680$

$\ldots|\ldots| \ldots|\ldots| \ldots|\ldots| \ldots|\ldots| \ldots|\ldots| \ldots|\ldots| \ldots|\ldots| \ldots \mid$ GGTAAGTCAGTTTTTTTTTTTGGCTAATAAAAACGGTAAAATCATGTTATTGATAAAAAA GGTAAGTCAGTTTTTTTTTTTGGCTAATAAAAACGGTAAAATCATGTTATTGATAAAAAA
1690
1700
1710
1720
1730
1740

$\ldots \ldots|\ldots| \ldots|\ldots| \ldots|\ldots| \ldots|\ldots| \ldots|\ldots| \ldots|\ldots| \ldots|\ldots|$ TTTGAAAACAGTAAAAGAAATATGACCATATTTGAAAATTTACCTAAAAACTATAATGAT TTTGAAAACAGTAAAAGAAATATGACCATATTTGAAAATTTACCTAAAAACTATAATGAT
1750
1760
1770
1780
1790
1800

$\ldots|\ldots| \ldots|\ldots| \ldots|\ldots| \ldots|\ldots| \ldots|\ldots| \ldots|\ldots| \ldots|\ldots| \ldots \mid$ GTAATAGTTTGATTTACTTACATTTAACTAATTTTTAAGGTCTGTTGATCGAACTCGTTA GTAATAGTTTGATTTACTTACATTTAACTAATTTTTAAGGTCTGTTGATCGAACTCGTTA

TCT
1810
1820
1830
1840
1850
1860

$\ldots|\ldots| \ldots|\ldots| \ldots|\ldots| \ldots|\ldots| \ldots|\ldots| \ldots|\ldots| \ldots|\ldots| \ldots|\ldots|$ ССTTGAACAATTAGTAGGACAACTCAAACCACTAAACCATTATACTTTAAGGATTTATGT CCTTGAACAATTAGTAGGACAACTCAAACCACTAAACCATTATACTTTAAGGATTTATGT

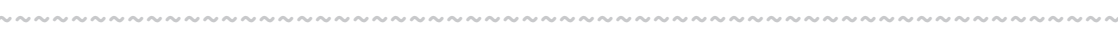
1870
1880
1890
1900
1910
1920

$\ldots \ldots|\ldots| \ldots|\ldots| \ldots|\ldots| \ldots|\ldots| \ldots|\ldots| \ldots|\ldots| \ldots|\ldots| \ldots \mid$ AAAATTTCAAATATATATAGTTTAGTAGATGCACTTATCATCACACTCACCAATTGGATG AAAATTTCAAATATATATAGTTTAGTAGATGCACTTATCATCACACTCACCAATTGGATG

$$
\begin{array}{llllll}
1930 & 1940 & 1950 & 1960 & 1970 & 1980
\end{array}
$$
$\ldots . \ldots|\ldots| \ldots|\ldots| \ldots|\ldots| \ldots|\ldots| \ldots|\ldots| \ldots|\ldots| \ldots|\ldots|$ TCAACACCTGGTTCTAGCTTTTTAATTACCAAAGTGAAAAAACTGACTTTTTCTAAAAAA TCAACACCTGGTTCTAGCTTTTTAATTACCAAAGTGAAAAAACTGACTTTTTCTAAAAAA

$$
\sim
$$

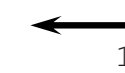
1990
2000
2010
2020
2030
2040

$\ldots|\ldots| \ldots|\ldots| \ldots|\ldots| \ldots|\ldots| \ldots|\ldots| \ldots|\ldots| \ldots|\ldots| \ldots \mid$ AATTCGTTCTAGATGGATGCTCTTCAAATTCGTTC TAAAAAAAACTG AATTCGTTCTAGATGGATGCTCTTCAAATTCGTTC TAAAAAAAACTG $\sim \sim \sim \sim \sim \sim$ TCTAGATGGATGCTCTTTAAAT C TTCGATATTTTTTATCCGTTTCGATAAT TCTAGATGGATGCTCTTTAAAT C TTCGATATTTTTTATCCGTTTCGATAAT

$$
\begin{gathered}
2050 \\
\ldots \ldots|\ldots| \ldots|\ldots| \ldots|\ldots| \ldots|\ldots| \ldots|\ldots| \ldots|\ldots| \ldots|\ldots| \ldots|\ldots|
\end{gathered}
$$

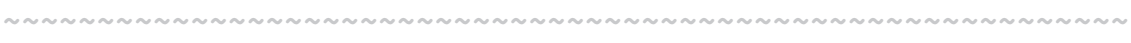

ATGGTAAGAATGAATGACGAATCGGTCAAGCTAATCTGTATATTAATCATTGTACTCATC ATGGTAAGAATGAATGACGAATCGGTCAAGCTAATCTGTATATTAA CATTGTACTCATC

$$
\begin{aligned}
& \begin{array}{llllll}
2110 & 2120 & 2130 & 2140 & 2150 & 2160
\end{array} \\
& \ldots|\ldots| \ldots|\ldots| \ldots|\ldots| \ldots|\ldots| \ldots|\ldots| \ldots|\ldots| \ldots|\ldots| \ldots \mid
\end{aligned}
$$


Col-0 VTC1 TAIR gDNA

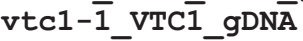
svt2 VT̄C1 gDNA

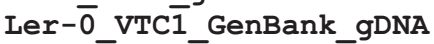

Col-0_VTC1_TAIR_gDNA vtc1-1̄ VTC $\overline{1}$ gDNA $\bar{A}$

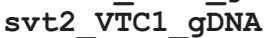

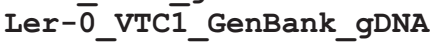

Col-0_VTC1_TAIR_gDNA vtc1-1̄ VTC $\overline{1}$ gDNA svt2 VTC1 gDNA

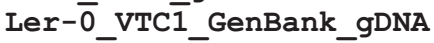

Col-0_VTC1_TAIR_gDNA vtc1-1̄_VTC $\overline{1}$ _gDNA svt2_vT 1 1_g $\overline{D N A}$

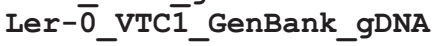

Col-0 VTC1 TAIR gDNA

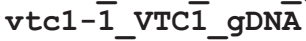
svt2 vT C1_gDNA

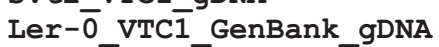

Col-0_VTC1_TAIR_gDNA vtc1-1̄ VTC $\overline{1}$ gDNA $\bar{A}$ svt2 VTC1 gDNA Ler-o_VTC1̄_GenBank_gDNA

Col-0_VTC1_TAIR_gDNA

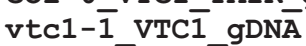
svt2 VTC1 gDNA

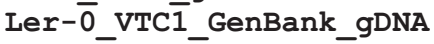

Col-0 VTC1 TAIR gDNA

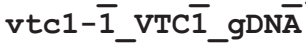
svt2 VTC1_gDNA

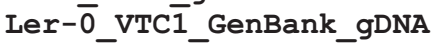

Col-0 VTC1 TAIR gDNA

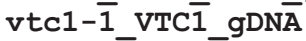
svt2 VTC1 gDNA Ler-o_VTC1̄_GenBank_gDNA
2170
$\ldots \ldots|\ldots| \ldots|\ldots| \ldots|\ldots| \ldots|\ldots| \ldots|\ldots| \ldots|\ldots| \ldots|\ldots| \ldots|\ldots| \ldots|\ldots| \ldots \mid$

СTACССTATATATAAAGTTCATCAGAGTTGTCGATCAGCAGTGACCACTACACATTCTTC СTACCCTAT AAAGTTCATCAGAGTTGTCGATCAGCAGTGACCACTACACATTCTTC

\section{$M M$}

2230

2240

2250

2260

2270

2280

$\ldots \ldots|\ldots| \ldots|\ldots| \ldots|\ldots| \ldots|\ldots| \ldots|\ldots| \ldots|\ldots| \ldots|\ldots| \ldots \mid$ ACATAATA ACATAATA ATACAGCTGAGTTAGGAATGTTAACAAAATAGTTTATGGGAGTATGTTTTATACATAATA ATACAGCTGAGTTAGGAATGTTAACAAAATAGTTTATGGGAGTATGTTTTATACATAATA

$2290 \quad 2300 \quad 2310 \quad 2320 \quad 2330 \quad 2340$ $\ldots|\ldots| \ldots|\ldots| \ldots|\ldots| \ldots|\ldots| \ldots|\ldots| \ldots|\ldots| \ldots|\ldots| \ldots \mid$ ССАTСCСTTTAAAAACACAGAATTTTTTTATCATCTCTGAAACAAATCATTTACAGTAG CСАTСССTTTAAAAACACAGAATTTTTTTTATCATCTCTGAAACAAATCATTTACAGTAG CСАTCCСTTTAAAAACACAGAATTTTCTTTATCATCTCCGAAACAAATCATTTACAGTAG CCATCCCTTTAAAAACACAGAATTTTCTTTATCATCTCCGAAACAAATCATTTACAGTAG $2350 \quad 2360 \quad \uparrow_{2370} \quad \uparrow_{2380} \quad 2390 \quad 2400$ $\ldots|\ldots| \ldots|\ldots| \ldots|\ldots| \ldots|\ldots| \ldots|\ldots| \ldots|\ldots| \ldots|\ldots| \ldots|\ldots|$ TAAATGT CAACACAACATTAATTCTGTTTGTTGTTGGCATTTACAATTGCAAAATCAT TAAATGT CAACACAACATTAATTCTGTTTGTTGTTGGCATTTACAATTGCAAAATCAT TAAATGTAAAAACACAACATTAATTCTGTTTGTTGTTGGCATTTACAATTGCAAAATCAT TAAATGTAAAAACACAACATTAATTCTGTTTGTTGTTGGCATTTACAATTGCAAAATCAT

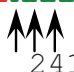

2420

2430

2440

2450

2460

$\ldots|\ldots| \ldots|\ldots| \ldots|\ldots| \ldots|\ldots| \ldots|\ldots| \ldots|\ldots| \ldots|\ldots| \ldots \mid$ TTTCTCATTTATTATTCGTATTTATTTTGTCAAGAACCCTTGTCTCTAAAATATTCATAG TTTCTCATTTATTATTCGTATTTATTTTGTCAAGAACCCTTGTCTCTAAAATATTCATAG TTTCTCATTTATTATTCGTATTTATTTTGTCAAGAACCCTTGTCTCTAAAATATTCATAG TTTCTCATTTATTATTCGTATTTATTTTGTCAAGAACCCTTGTCTCTAAAATATTCATAG

$$
2470 \quad 2480 \quad 2490 \quad 2500 \quad 2510 \quad 2520
$$

$\ldots|\ldots| \ldots|\ldots| \ldots|\ldots| \ldots|\ldots| \ldots|\ldots| \ldots|\ldots| \ldots|\ldots| \ldots \mid$ AAAAAGAAAAGAGCCATTAATTAATGGCTTGAAGAAAGATTGGTGTATAAGCGTCTACGT AAAAAGAAAAGAGCCATTAATTAATGGCTTGAAGAAAGATTGGTGTATAAGCGTCTACGT AAAAA AAAAGAGCCATTAATTAATGGCTTGAAGAAAGATTGGTGTATAAGCGTCTACGT AAAAA AAAAGAGCCATTAATTAATGGCTTGAAGAAAGATTGGTGTATAAGCGTCTACGT $\uparrow$

$$
\begin{gathered}
2530 \\
\ldots .|\ldots| \ldots|\ldots| \ldots|\ldots| \ldots|\ldots| \ldots|\ldots| \ldots|\ldots| \ldots|\ldots| \ldots|\ldots| \ldots \mid
\end{gathered}
$$
GACCTTTAATTAATTTACTTCCCCCAAAAAAGTCAACATTCAACATGTGAATAAAAA TC GACCTTTAA TTAATTTACTTCCCCCAAAAAAGTCAACATTCAACATGTGAATAAAAA TC GACСTTTAАTTAАTTTACTTCCCCAAAAAAAGTCAACATTCAACATGTGAATAAAAA TC GACCTTTAATTAATTTACTTCCCCAAAAAAAGTCAACATTCAACATGTGAATAAAAAATC

$$
2590 \quad 2600{ }^{\uparrow} 2610 \quad 2620 \quad 2630 \quad 2640
$$

$\ldots|\ldots| \ldots|\ldots| \ldots|\ldots| \ldots|\ldots| \ldots|\ldots| \ldots|\ldots| \ldots|\ldots|$ AATATTGGTTTCTAAGTAAGTAAGTACCATATTATTAAATTATTTATTTTGGTAAATACG AATATTGGTTTCTAAGTAAGTAAGTACCATATTATTAAATTATTTATTTTGGTAAATACG AATATTGGTTTCTAAGTAAGTAAGTACCATATTATTAAATTATTTATTTTGGTAAATACG AATATTGGTTTCTAAGTAAGTAAGTACCATATTATTAAATTATTTATTTTGGTAAATACG

$$
2650 \quad 2660 \quad 2670 \quad 2680 \quad 2690 \quad 2700
$$

$\ldots|\ldots| \ldots|\ldots| \ldots|\ldots| \ldots|\ldots| \ldots|\ldots| \ldots|\ldots| \ldots|\ldots| \ldots \mid$ САСТСAАTTTTTCTCTCAACGGTG TATATAAACAAAAGGAGTCTCCTTTGGAAAAA САСТСААТTTTTСТСТСАACGGTG TATATAAACAAAAGGAGTCTCСTTTGGAAAAA САСTCAATTTTTCTCTCAACGGTGGGTGTATATAAACAAAAGGAGTCTCCTTTGGAAAAA САСTCAATTTTTCTCTCAACGGTGGGTGTATATAAACAAAAGGAGTCTCCTTTGGAAAAA 
Col-0 VTC1 TAIR gDNA vtc1-̄̄ VTC $\overline{1}$ gDNA svt2 VTC1 gDNA Ler-ō_VTC $\overline{1}$ _GenBank_gDNA

COI-0 VTC1 TAIR_gDNA

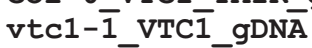
svt2 VTC1 gDNA

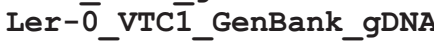

Col-0 VTC1 TAIR gDNA

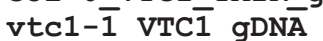
svt2_vT̄C1_gDNA Ler-ō_VTC $\overline{1}$ _GenBank_gDNA

Col-0_VTC1_TAIR_gDNA

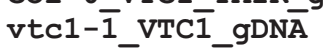

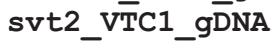

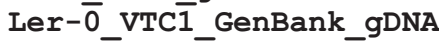

Col-0_VTC1_TAIR_gDNA

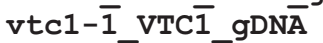
svt2_VTC1_gDNA Ler-ō_VTC $\overline{1}$ _GenBank_gDNA

Col-0_VTC1_TAIR_gDNA

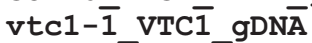
svt2 VTC1_gDNA Ler-0_VTC1̄_GenBank_gDNA

Col-0_VTC1_TAIR_gDNA vtc1-1__VTC1__gDNA svt2 VTC1 gD̄NA

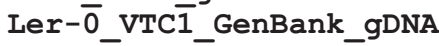

Col-0_VTC1_TAIR_gDNA

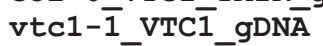
svt2_VTC1_ḡ $\overline{\mathrm{DNA}}$

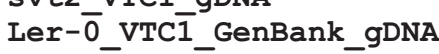

COI-0 VTC1 TAIR gDNA

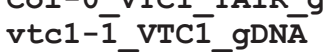
svt2 VT̄C1 gDNA Ler-̄o_VTC $\overline{1}$ GenBank_gDNA
2710
$\ldots .|\ldots| \ldots|\ldots| \ldots|\ldots| \ldots|\ldots| \ldots|\ldots| \ldots|\ldots| \ldots|\ldots| \ldots|\ldots| \ldots \mid$ ACTTGCCTATCATTTTGCCAACGAACGTTCTTTCTTCTTAATCACAGCTCAGCCTGACGC ACTTGCCTATCATTTTGCCAACGAACGTTCTTTCTTCTTAATCACAGCTCAGCCTGACGC ACTTGCCTATCATTTTGCCAACGAACGTTCTTTCTTCTTAATCACAGCTCAGCCTGACGC ACTTGCCTATCATTTTGCCAACGAACGTTCTTTCTTCTTAATCACAGCTCAGCCT ACGC

$$
2770 \quad 2780 \quad 2790 \quad 2800 \quad 2810 \quad 2820
$$

$\ldots|\ldots| \ldots|\ldots| \ldots|\ldots| \ldots|\ldots| \ldots|\ldots| \ldots|\ldots| \ldots|\ldots|$ AACCGCTCAGGCTGATCTCTTCCAATTTACAGCCATTTCCCAGCTCAGATCTCTGATCCG AACCGCTCAGGCTGATCTCTTCCAATTTACAGCCATTTCCCAGCTCAGATCTCTGATCCG AACCGCTCAGGCTGATCTCTTCCAATTTACAGCCATTTCCCAGCTCAGATCTCTGATCCG AACCGCTCAGGCTGATCTCTTCCAATTTACAGCCATTTCCCAGCTCAGATCTCTGATCCG

$$
2830 \quad 2840 \quad 2850 \quad 2860 \quad 2870 \quad 2880
$$

$\ldots|\ldots| \ldots|\ldots| \ldots|\ldots| \ldots|\ldots| \ldots|\ldots| \ldots|\ldots| \ldots|\ldots| \ldots \mid$ GTGAGATCTCTCTCAAGGTAATGCCССTGCAATTTTGCTTACTTCTCTGGTTGTGATATG GTGAGATCTCTCTCAAGGTAATGCCCСTGCAATTTTGCTTACTTCTCTGGTTGTGATATG GTGAGATCTCTCTCAAGGTAATGCCCСTGCAATTTTGCTTACTTCTCTGGTTGTGATATG GTGAGATCTCTCTCAAGGTAATGCCССTGCAATTTTGCTTACTTCTCTGGTTGTGATATG

$$
2890 \quad 2900 \quad 2910 \quad 2920 \quad 293 p \quad 2940
$$
$\ldots|\ldots| \ldots|\ldots| \ldots|\ldots| \ldots|\ldots| \ldots|\ldots| \ldots|\ldots \ldots| \ldots \ldots|\ldots|$ CATGTTCTTCGAATTTTCATCGTTTGTGATTTGAATTCTCATTTTGTATTTGCTGTTGTT CATGTTCTTCGAATTTTCATCGTTTGTGATTTGAATCTCATTTTGTATTTGCTGTTGTT CATGTTCTTCGAATTTTCATCGTTTGTGATTTGAATTCTCATTTTGTATTTGCTGTTGTT CATGTTCTTCGAATTTTCATCGTTTGTGATTTGAATTCTCATTTTGTATTT CTGTTGTT

$$
29502960 \quad 2970 \quad 2980 \downarrow 2990 \quad 3000
$$

$\ldots|\ldots| \ldots|\ldots| \ldots|\ldots| \ldots|\ldots| \ldots|\ldots| \ldots|\ldots| \ldots|\ldots| \ldots \mid$ GGTTTTTAATTCGATTTTCCGGAACAGATTATGGGATTT GTATTCGAATCTTCGATTTG GGTTTTTAATTCGATTTTCCGGAACAGATTATGGGATTT GTATTCGAATCTTCGATTTG GGTTTTTAATTCGATTTTCCGGAACAGGTTATGGGGATTTGTATTCGAATCTTCGATTTG GGTTTTTAATTCGATTTTCCGGAACAGGTTATGGGGATTTGTAT CGAATCTTCGATTTG

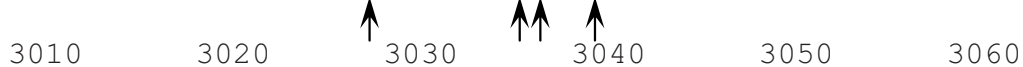

$\ldots|\ldots| \ldots|\ldots| \ldots|\ldots| \ldots|\ldots| \ldots|\ldots| \ldots|\ldots| \ldots|\ldots| \ldots \mid$ ATGACATAATGTCCCAGCCTTTTATGTTTAATCTTGAAATGATGGACTTTTATCCGATCT ATGACATAATGTCCCAGCCTTTTATGTTTAATCTTGAAATGATGGACTTTTATCCGATCT ATGACATAATGTCCCAGCCATTTATGTTTAATCTTGAAATGATGGACTTTAATCCGATCT ATGACATAATGTCCCAGCCATTTATGTTTAATCTTGAAATGATGGACTTTAATCCGATCT

$$
\begin{array}{llllll} 
& \uparrow & & & & \\
3070 & 3080 & 3090 & 3100 & 3110 & 3120
\end{array}
$$

$\ldots|\ldots| \ldots|\ldots| \ldots|\ldots| \ldots|\ldots| \ldots|\ldots| \ldots|\ldots| \ldots|\ldots|$ GGGTTTAAAGCTGGAATTTTGATTGTGGGTACTATTAGGTTTCATTGATTTATTGCTTGG GGGTTTAAAGCTGGAATTTTGATTGTGGGTACTATTAGGTTTCATTGATTTATTGCTTGG GGGTTTAAAGCTGGAATTTTGATTGTGGGTACTATTAGGTTTCATTGATTTATTGCTTGG GGGTTTAAAGCTGGAATTTTGATTGTGGGTACTATTAGGTTTCATTGATTTATTGCTTGG

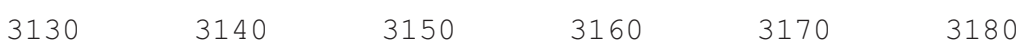

$\ldots|\ldots| \ldots|\ldots| \ldots|\ldots| \ldots|\ldots| \ldots|\ldots| \ldots|\ldots| \ldots|\ldots| \ldots \mid$ TCCAACATTTTTAGCAGCTGGTATTGAGCTCTTGTTGTCTGAATTTTGGAAAGAACTATT TCCAACATTTTTAGCAGCTGGTATTGAGCTCTTGTTGTCTGAATTTTGGAAAGAACTATT TCCAACATTTTTAGCAGCTGGTATTGAGCTCTTGTTGTCTGAATTTTGGAAAGAACTATT TCCAACATTTTTAGCAGCTGGTATTGAGCTCTTGTTGTCTGAATTTTGGAAAGAACTATT

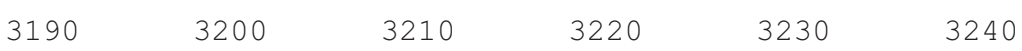

$\ldots|\ldots| \ldots|\ldots| \ldots|\ldots| \ldots|\ldots| \ldots|\ldots| \ldots|\ldots| \ldots|\ldots| \ldots \mid$ TTTGTTGTATCGTTTTGATTTATTTGGATCTGAATTCATTCACCTTTTTCTCTGATTATT TTTGTTGTATCGTTTTGATTTATTTGGATCTGAATTCATTCACCTTTTTCTCTGATTATT TTTGTTGTATCGTTTTGATTATTTGGATCTGAATCATTCACCTTTTTCTCTGATTATT TTTGTTGTATCGTTTTGATTAATTGGATCTGAATTCATTCACCTTTTTCTCTGATTATT 
Col-0 VTC1 TAIR GDNA

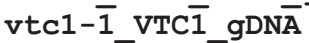
svt2 VTC1 gDNA

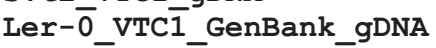

Col-0_VTC1_TAIR_gDNA

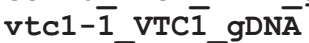
svt2 VTC1 gDNA

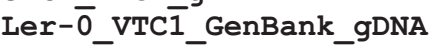

CO1-0_VTC1 TAIR gDNA vtc1-1̄_VTC $\overline{1}$ gDNĀ svt2 VTC1 gDNA

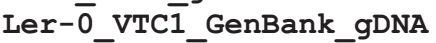

Col-0_VTC1_TAIR_gDNA

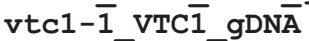
svt2_VTC1_gDNA

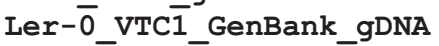

Col-0 VTC1 TAIR gDNA vtc1-1̄ VTC $\overline{1}$ gDNA svt2 VTC1_gDNA Ler-̄o_VTC $\overline{1}$ _GenBank_gDNA

Col-0_VTC1_TAIR_gDNA

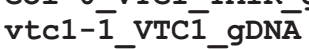
svt2 VTC1 gDNA

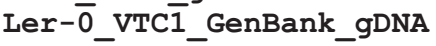

Col-0 VTC1 TAIR gDNA vtc1-1̄_VTC1̄_gDNA svt2 VTC1 gDNA

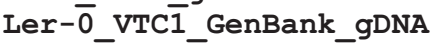

Col-0 VTC1 TAIR gDNA vtc1-̄̄ VTC $\overline{1}$ gDNA svt2 VTC1 gDNA

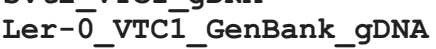

Col-0_VTC1_TAIR_gDNA

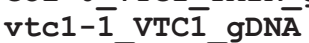
svt2 VTC1 gDNA

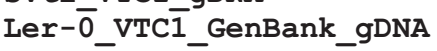

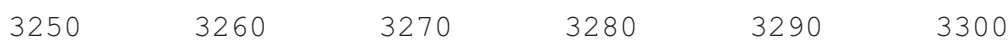

$\ldots|\ldots| \ldots|\ldots| \ldots|\ldots| \ldots|\ldots| \ldots|\ldots| \ldots|\ldots| \ldots|\ldots| \ldots \mid$ GTTTTGTGTCGGTTGCATCCACTTTGATTAGATCTGAATGAATCATTTTTTTATGTGCTC GTTTTGTGTCGGTTGCATCCACTTTGATTAGATCTGAATGAATCATTTTTTTATGTGCTC GTTTTGTGTCGGTTGCATCCACTTTGATTAGATCTGAATGAATCATTTTTTTATGTGCTC GTTTTGTGTCGGTTGCATCCACTTTGATTAGATCTGAATGAATCATTTTTTTATGTGCTC

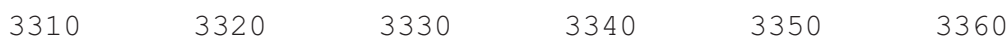
$\ldots|\ldots| \ldots|\ldots| \ldots|\ldots| \ldots|\ldots| \ldots|\ldots| \ldots|\ldots| \ldots|\ldots| \ldots \mid$ AAGTTATTGTATGGATTGTTCTGTTTCTAGCATGTTTTGGTTAGACATTGTTAAGATCTG AAGTTATTGTATGGATTGTTCTGTTTCTAGCATGTTTTGGTTAGACATTGTTAAGATCTG AAGTTATTGTATGGATTGTTCTGTTTCTAGCATGTTTTGGTTAGACATTGTTAAGATCTG AAGTTATTGTATGGATTGTTCTGTTCTAGCATGTTTTGGTTAGACATTGTTAAGATCTG

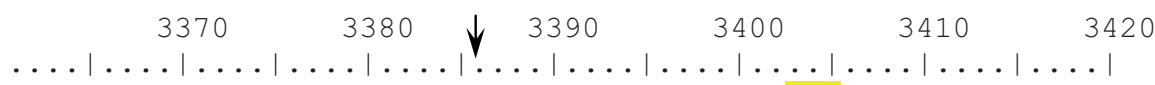
ACGTTTGCATTTTCAGGAAAAGGA TTAGAGCATCATCAAGATGAAGGCACTCATTCTT ACGTTTGCATTTTCAGGAAAAGGAG TTAGAGCATCATCAAGATGAAGGCACTCATTCTT ACGTTTGCATTTTCAGGAAAAGGAG TTAGAGCATCATCAAGATGAAGGCACTCATTCTT ACGTTTGCATTTTCAGGAAAAGGAGCTTAGAGCATCATCAAGATGAAGGCACTCATTCTT
3430
3440
3450
3460
3470
3480

$\ldots|\ldots| \ldots|\ldots| \ldots|\ldots| \ldots|\ldots| \ldots|\ldots| \ldots|\ldots| \ldots|\ldots| \ldots \mid$ GTTGGAGGCTTCGGCACTCGCTTGAGACCATTGACTCTCAGTTTCCCAAAGCCCCTTGTT GTTGGAGGCTTCGGCACTCGCTTGAGACCATTGACTCTCAGTTTCTCAAAGCCCCTTGTT GTTGGAGGCTTCGGCACTCGCTTGAGACCATTGACTCTCAGTTTCCCAAAGCCCCTTGTT GTTGGAGGCTTCGGCACTCGCTTGAGACCATTGACTCTCAGTTTCCCAAAGCCCСTTGTT

$$
3490 \quad 3500 \quad 3510 \quad 3520 \quad 3530 \quad 3540
$$

$\ldots|\ldots| \ldots|\ldots| \ldots|\ldots| \ldots|\ldots| \ldots|\ldots| \ldots|\ldots| \ldots|\ldots| \ldots \mid$ GATTTTGCTAATAAACCCATGATCCTTCATCAGGTAATCTATCTTAAATTTGCCGCTTTA GATTTTGCTAATAAACCCATGATCCTTCATCAGGTAATCTATCTTAAATTTGCCGCTTTA GATTTTGCTAATAAACCCATGATCCTTCATCAGGTAATCTATCTTAAATTTGCCGCTTTA GATTTTGCTAATAAACCCATGATCCTTCATCAGGTAATCTATCTTAAATTTGCCGCTTTA

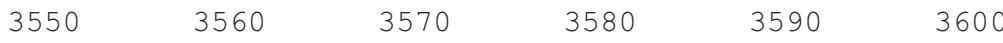
$\ldots|\ldots| \ldots|\ldots| \ldots|\ldots| \ldots|\ldots| \ldots|\ldots| \ldots|\ldots| \ldots|\ldots| \ldots \mid$ GTCTGCCAGTTCTTACCTATGCCTATGTTTGAACCGAGGCATGTTTTCTTGTAGATAGAG GTCTGCCAGTTCTTACCTATGCCTATGTTTGAACCGAGGCATGTTTTCTTGTAGATAGAG GTCTGCCAGTTCTTACCTATGCCTATGTTTGAACCGAGGCATGTTTTCTTGTAGATAGAG GTCTGCCAGTTCTTACCTATGCCTATGTTTGAACCGAGGCATGTTTTCTTGTAGATAGAG

$$
\begin{array}{lllll}
3610 & 3620 \quad 3630 & 3640 & 3650 & 3660
\end{array}
$$

$\ldots|\ldots| \ldots|\ldots| \ldots|\ldots| \ldots|\ldots| \ldots|\ldots| \ldots|\ldots| \ldots|\ldots| \ldots \mid$ GCTCTTAAGGCAGTTGGAGTTGATGAAGTGGTTTTGGCCATCAATTATCAGCCAGAGGTA GCTCTTAAGGCAGTTGGAGTTGATGAAGTGGTTTTGGCCATCAATTATCAGCCAGAGGTA GCTCTTAAGGCAGTTGGAGTTGATGAAGTGGTTTTGGCCATCAATTATCAGCCAGAGGTA GCTCTTAAGGCAGTTGGAGTTGATGAAGTGGTTTTGGCCATCAATTATCAGCCAGAGGTA

$\begin{array}{lllll}3670 & 3680 & 3690 & 3700 & 3710\end{array}$

$\ldots|\ldots| \ldots|\ldots| \ldots|\ldots| \ldots|\ldots| \ldots|\ldots| \ldots|\ldots| \ldots|\ldots| \ldots|\ldots|$ AGATACTAATCTCTCTTAACTTTTTTTTTTGCAGCTATTTTCTGTTTACATATGTTTGTA AGATACTAATCTCTCTTAACTTTTTTTTTTGCAGCTATTTTCTGTTTACATATGTTTGTA AGATACTAATCTCTCTTAACTTTTTTTTTGCAGCTATTTTCTGTTTACATATGTTTGTA AGATACTAATCTCTCTTAACTTTTTTTTTTGCAGCTATTTTCTGTTTACATATGTTTGTA

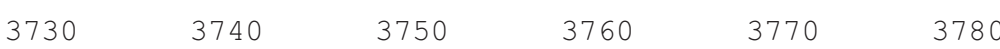
$\ldots|\ldots| \ldots|\ldots| \ldots|\ldots| \ldots|\ldots| \ldots|\ldots| \ldots|\ldots| \ldots|\ldots| \ldots \mid$ TTTACCATTTGCTCTGTTTCGACAGGTGATGCTGAACTTCTTGAAGGACTTTGAGACCAA TTTACCATTTGCTCTGTTTCGACAGGTGATGCTGAACTTCTTGAAGGACTTTGAGACCAA TTTACCATTTGCTCTGTTTCGACAGGTGATGCTGAACTTCTTGAAGGACTTTGAAACCAA TTTACCATTTGCTCTGTTTCGACAGGTGATGCTGAACTTCTTGAAGGACTTTGAGACCAA 
Col-0_VTC1_TAIR_gDNA vtc1-1̄VTC $\overline{1}$ gDNA svt2 VTC1 gDNA

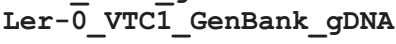

Col-0 VTC1 TAIR gDNA

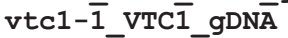
svt2 VTC1 gDNA

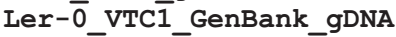

Col-0 VTC1 TAIR gDNA vtc1- $\overline{1}$ VTC $\overline{1}$ gDNA svt2_VTC1_gDNA Ler-ōVTC1̄_GenBank_gDNA

COI-0_VTC1_TAIR_gDNA vtc1-1̄_VTC $\overline{1}$ _gDNA svt2 VTC1 gDNA Ler-0̄VTC1'_GenBank_gDNA

Col-0 VTC1 TAIR gDNA

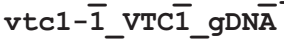
svt2 VTC1 gDNA

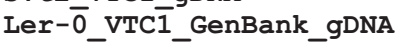

Col-0 VTC1 TAIR gDNA vtc1-1̄ VTC $\overline{1}$ _gDNA svt2_vT̄C1_gDNA Ler-̄o_VTC1__GenBank_gDNA

Col-0 VTC1 TAIR GDNA

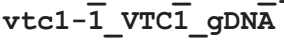
svt2 VT̄C1 gDNA

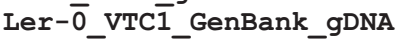

Col-0 VTC1 TAIR gDNA vtc1-1̄ VTC $\overline{1}$ _gDNA svt2 VTC1 gDNA

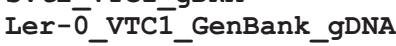

Col-0_VTC1_TAIR_gDNA vtc1- $\overline{1}$ VTC $\overline{1}$ gDNA svt2 VTC1 gDNA Ler-o_VTCí1_GenBank_gDNA

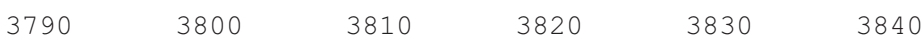
$\ldots|\ldots| \ldots|\ldots| \ldots|\ldots| \ldots|\ldots| \ldots|\ldots| \ldots|\ldots| \ldots|\ldots|$ GCTGGAAATCAAAATCACTTGCTCACAAGAGACCGAGCCACTAGGTACCGCTGGTCCTCT GCTGGAAATCAAAATCACTTGCTCACAAGAGACCGAGCCACTAGGTACCGCTGGTCCTCT GCTGGAAATCAAAATCACTTGCTCACAAAAAACCGAGCCACTAGGTACCGCTGGTCCTCT GCTGGAAATCAAAATCACTTGCTCACAAGAGACCGAGCCACTAGGTACCGCTGGTCCTCT

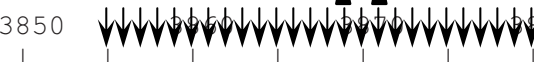

$\ldots|\ldots| \ldots|\ldots| \ldots|\ldots| \ldots \mid \ldots \ldots$ GGCTCTAGCGAGAG GGCTCTAGCGAGAG

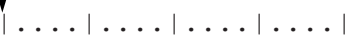
ACAATTGCTTGATGGATCT ACAAATTGCTTGATGGATCT GGCTCTAGCGAGAGGTTGGCGTAATCATGGTCATAGCTAGACAAATTGCTTGATGGATCT
3910
3920
3930
3940
3950
$\downarrow$
3960

$\ldots . \ldots|\ldots| \ldots|\ldots| \ldots|\ldots| \ldots|\ldots| \ldots|\ldots| \ldots|\ldots| \ldots|\ldots|$ GGAGAGCCCTTCTTTGTTCTTAACAGTGATGTGATTAGTGAGTACCCTCTTAAAGAAATG GGAGAGCCCTTCTTTGTTCTTAACAGTGATGTGATTAGTGAGTACCCTCTTAAAGAAATG GGAAAGCCCTTCTTTGTTCTTAACAGTGATGTGATTAGTGAGTACCCTCTTAAAGAAATG GGAGAGCCCTTCTTTGTTCTTAACAGTGATGTGATTAGTGAGTACCCTCTTAAAGAAATC

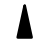
3970
3980
3990
4000
$4010 \downarrow 4020$

$\ldots . \ldots|\ldots| \ldots|\ldots| \ldots|\ldots| \ldots|\ldots| \ldots|\ldots| \ldots|\ldots| \ldots \mid \ldots$ CTTGAGTTTCACAAATCTCACGGTGGGGAAGCCTCCATAATGGTAACAAAGGTGAGATTA CTTGAGTTTCACAAATCTCACGGTGGGGAAGCCTCCATAATGGTAACAAAGGTGAGATTA CTTGAGTTTCACAAATCTCACGGTGGGGAAGCCTCCATAATGGTAACAAAGGTGAGATTA CTTGAGTTTCACAAATCTCACGGTGGGGAAGCCTCCATAATGGTAACAAAGGTG GATTA

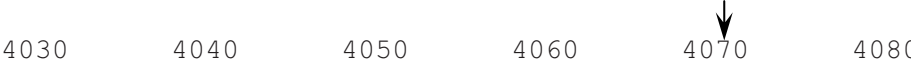

$\ldots|\ldots| \ldots|\ldots| \ldots|\ldots| \ldots|\ldots| \ldots|\ldots| \ldots|\ldots| \ldots|\ldots| \ldots \mid$ TCGAAACATAATACTCTCCAGTTACGAGATAAGTACGTTATTCATCTAATGTGGACTTGC TCGAAACATAATACTCTCCAGTTACGAGATAAGTACGTTATTCATCTAATGTGGACTTGC TCGAAACATAATACTCTCCAGTTACGAGATAAGTACGTTATTCATCTAATGTGGACTTGC TCGAAACATAATACTCTCCAGTTACGAGATAAGTACGTTATTCATCTAATCTGGACTTGC
4090
4100
4110
4120
4130
4140

$\ldots \ldots|\ldots| \ldots|\ldots| \ldots|\ldots| \ldots|\ldots| \ldots|\ldots| \ldots \mid \ldots \ldots$ ATGTATTGGTTATATAGGTGGATGAACCGTCGAAATATGGAGTGGTTGTTATGGAAGAAA ATGTATTGGTTATATAGGTGGATGAACCGTCGAAATATGGAGTGGTTGTTATGGAAGAAA ATGTATTGGTTATATAGGTGGATGAACCGTCGAAATATGGAGTGGTTGTTATGGAAGAAA ATGTATTGGTTATATAGGTGGATGAACCGTCGAAATATGGAGTGGTTGTTATGGAAGAAA
4150
4160
4170
4180
4190
4200

$\ldots|\ldots| \ldots|\ldots| \ldots|\ldots| \ldots|\ldots| \ldots|\ldots| \ldots|\ldots| \ldots|\ldots| \ldots$ GCACTGGAAGA GTGGAGAAGTTTGTGGAAAAGCCAAAACTGTATGTAGGTAACAAGATCA GCACTGGAAGAGTGGAGAAGTTTGTGGAAAAGCCAAAACTGTATGTAGGTAACAAGATCA GCACTGGAAGAGTGGAGAAGTTTGTGGAAAAGCCAAAACTGTATGTAGGTAACAAGATCA GCACTGGAAGAGTGGAGAAGTTTGTGGAAAAGCCAAAACTGTATGTAGGTAACAAGATCA

$\begin{array}{rrrrr}4210 & 4220 & 4230 & 4240 & 4250\end{array} 4260$ $\ldots . \ldots|\ldots| \ldots|\ldots| \ldots|\ldots| \ldots|\ldots| \ldots|\ldots| \ldots|\ldots| \ldots|\ldots|$ ACGCTGGGATTTATCTTCTGAACCCATCTGTTCTTGATAAGATTGAGCTAAGACCGACTT ACGCTGGGATTTATCTTCTGAACCCATCTGTTCTTGATAAGATTGAGCTAAGACCGACTT ACGCTGGGATTTATCTTCTGAACCCATCTGTTCTTGATAAGATTGAGCTAAGACCGACTT ACGCTGGGATTTATCTTCTGAACCCATCTGTTCTTGATAAGATTGAGCTAAGACCGACTT

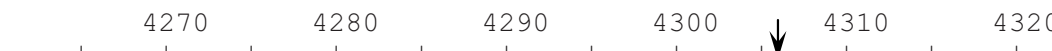
$\ldots|\ldots| \ldots|\ldots| \ldots|\ldots| \ldots|\ldots| \ldots|\ldots| \ldots|\ldots| \ldots|\ldots|$ CAATCGAAAAAGAGACTTTCCCTAAGATTGCAGCAGCGCAAGGGCTCTATGCTATGGTGC CAATCGAAAAAGAGACTTTCCCTAAGATTGCAGCAGCGCAAGGGCTCTATGCTATGGTGC CAATCGAAAAAGAGACTTTCCCTAAGATTGCAGCAGCGCAAGGGCTCTATGCTATGGTGC CAATCGAAAAAGAGACTTTCCCTAAGATTGCAGCAGCGCAAGGGCACTATGCTATGGTGC 
Col-0_VTC1_TAIR_gDNA
vtc1-1VTC 1 -gDNA
svt2_VTC1_gDNA
Ler-0_VTC1_GenBank_gDNA

Col-0 VTC1 TAIR gDNA

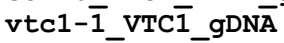
svt2 VTC1 gDNA

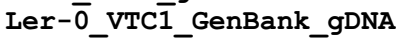

Col-0_VTC1 TAIR gDNA vtc1-1̄_VTC $\overline{1}$ gDNĀ svt2_VTC1_gDNA

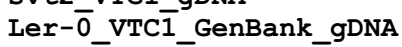

Col-0_VTC1_TAIR_gDNA vtc1-̄̄ VTC $\overline{1}$ gDNA svt2 VTC1 gDNA Ler-0_VTC1__GenBank_gDNA

Col-0_VTC1_TAIR_gDNA

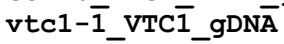
svt2 VTC1 gDNA

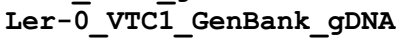

Col-0_VTC1_TAIR_gDNA

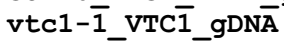
svt2 VTC1 gDNA Ler-ōVTC1̄_GenBank_gDNA

Col-0_VTC1_TAIR_gDNA vtc1-̄̄_VTC $\overline{1}$ gDNA svt2 VT̄C1 gDNA Ler-0_VTC1'_GenBank_gDNA

Col-0 VTC1 TAIR gDNA

vtc1-1̄_vTC $\overline{1}$ gDNA svt2 VTC1 gDNA

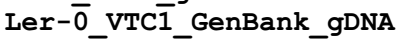

Col-0_VTC1_TAIR_gDNA vtc1- $\overline{1}$ VTC $\overline{1}$ gDNA svt2 vT̄C1 gDNA

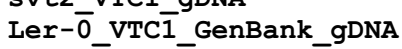

$\begin{array}{rrrrrr}4330 & 4340 & 4350 & 4360 & 4370 & 4380\end{array}$

$\ldots|\ldots| \ldots|\ldots| \ldots|\ldots| \ldots|\ldots| \ldots|\ldots| \ldots|\ldots| \ldots|\ldots|$

TACCAGGGTTTTGGATGGACATTGGGCAACCCCGTGACTACATAACGGGTTTGAGACTCT TACCAGGGTTTTGGATGGACATTGGGCAACCCCGTGACTACATAACGGGTTTGAGACTCT TACCAGGGTTTTGGATGGACATTGGGCAACCCCGTGACTACATAACGGGTTTGAGACTCT TACCAGGGTTTTGGATGGACATTGGGCAACCCCGTGACTACATAACGGGTTTGAGACTCT

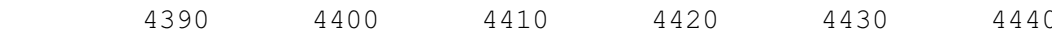

$\ldots|\ldots| \ldots|\ldots| \ldots|\ldots| \ldots|\ldots| \ldots|\ldots| \ldots|\ldots| \ldots|\ldots|$ ACTTAGACTCCCTTAGGAAGAAATCTCCTGCCAAATTAACCAGTGGGCCACACATAGTTG ACTTAGACTCCCTTAGGAAGAAATCTCCTGCCAAATTAACCAGTGGGCCACACATAGTTG ACTTAGACTCCCTTAGGAAGAAATCTCCTGCCAAATTAACCAGTGGGCCACACATAGTTG ACTTAGACTCCCTTAGGAAGAAATCTCCTGCCAAATTAACCAGTGGGCCACACATAGTTG

$\begin{array}{rrrrr}4450 & 4460 \quad 4470 \quad 4480 \quad 4490 \quad 4500\end{array}$ $\ldots . \ldots .|\ldots| \ldots|\ldots| \ldots|\ldots| \ldots|\ldots| \ldots|\ldots| \ldots|\ldots| \ldots|$. GGAATGTTCTTGTTGACGAAACCGCTACAATTGGGGAAGGATGTTTGATTGGACCAGACG GGAATGTTCTTGTTGACGAAACCGCTACAATTGGGGAAGGATGTTTGATTGGACCAGACG GGAATGTTCTTGTTGACGAAACCGCTACAATTGGGGAAGGATGTTTGATTGGACCAGACG GGAATGTTCTTGTTGACGAAACCGCTACAATTGGGGAAGGATGTTTGATTGGACCAGACG
4510
4520
4530
4540
4550
4560

$\ldots .|\ldots| \ldots|\ldots| \ldots|\ldots| \ldots|\ldots| \ldots|\ldots| \ldots|\ldots| \ldots|\ldots|$ TTGCCATTGGTCCAGGCTGCATTGTTGAGTCAGGAGTCAGACTCTCCCGATGCACGGTCA TTGCCATTGGTCCAGGCTGCATTGTTGAGTCAGGAGTCAGACTCTCCCGATGCACGGTCA TTGCCATTGGTCCAGGCTGCATTGTTGAGTCAGGAGTCAGACTCTCCCGATGCACGGTCA TTGCCATTGGTCCAGGCTGCATTGTTGAGTCAGGAGTCAGACTCTCCCGATGCACGGTCA

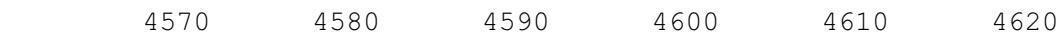
$\ldots \ldots|\ldots| \ldots|\ldots| \ldots|\ldots| \ldots|\ldots| \ldots|\ldots| \ldots|\ldots| \ldots|\ldots|$ TGCGTGGAGTCCGCATCAAGAAGCATGCGTGTATCTCGAGCAGTATCATCGGGTGGCACT TGCGTGGAGTCCGCATCAAGAAGCATGCGTGTATCTCGAGCAGTATCATCGGGTGGCACT TGCGTGGAGTCCGCATCAAGAAGCATGCGTGTATCTCGAGCAGTATCATCGGGTGGCACT TGCGTGGAGTCCGCATCAAGAAGCATGCGTGTATCTCGAGCAGTATCATCGGGTGGCACT

$\begin{array}{rrrrrr}4630 & 4640 & 4650 & 4660 & 4670 & 4680\end{array}$ $\ldots . \ldots .|\ldots| \ldots|\ldots| \ldots|\ldots| \ldots|\ldots| \ldots .|\ldots| \ldots|\ldots \ldots| \ldots \mid$ CAACGGTTGGTCAATGGGCCAGGATCGAGAACATGACGATCCTCGGTGAGGATGTTCATG CAACGGTTGGTCAATGGGCCAGGATCGAGAACATGACGATCCTCGGTGAGGATGTTCATG CAACGGTTGGTCAATGGGCCAGGATCGAGAACATGACGATCCTCGGTGAGGATGTTCATG CAACGGTTGGTCAATGGGCCAGGATCGAGAACATGACGATCCTCGGTGAGGATGTTCATG
4690
4700
4710
4720
4730
4740

$\ldots . \ldots|\ldots| \ldots|\ldots| \ldots|\ldots| \ldots|\ldots| \ldots|\ldots| \ldots|\ldots| \ldots$ TGAGCGATGAGATCTATAGCAATGGAGGAGTTGTTTTGCCACACAAGGAGATCAAATCAA TGAGCGATGAGATCTATAGCAATGGAGGAGTTGTTTTGCCACACAAGGAGATCAAATCAA TGAGCGATGAGATCTATAGCAATGGAGGAGTTGTTTTGCCACACAAGGAGATCAAATCAA TGAGCGATGAGATCTATAGCAATGGAGGAGTTGTTTTGCCACACAAGGAGATCAAATCAA

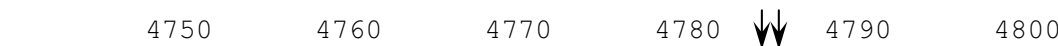
$\ldots|\ldots| \ldots|\ldots| \ldots|\ldots| \ldots|\ldots| \ldots|\ldots| \ldots|\ldots| \ldots|\ldots|$ ACATCTTGAAGCCAGAGATAGTGATGTGAAAATGAGATATTATA TGTGCAACTTTTTT ACATCTTGAAGCCAGAGATAGTGATGTGAAAATGAGATATTATA TGTGCAACTTTTTT ACATCTTGAAGCCAGAGATAGTGATGTGAAAATGAGATATTATA TGTGCAACTTTTTT ACATCTTGAAGCCAGAGATAGTGATGTGAAAATGAGATATTATATATGTGCAACTTTTTT

$$
\begin{array}{llllll}
4810 & 4820 \quad 4830 & 4840 \quad 4850 \quad 4860
\end{array}
$$
$\ldots .|\ldots| \ldots|\ldots| \ldots|\ldots| \ldots|\ldots| \ldots|\ldots| \ldots|\ldots| \ldots|\ldots|$ TTTTTTTTTT GTGTCCTTTCTTCAACTTTGAAATCGCTTTCGTAATTCTTAATGGCTTT TTTTTTTTTT GTGTCCTTTCTTCAACTTTGAAATCGCTTTCGTAATTCTTAATGGCTTT TTTTTTTTTT

TTTTTTTTTTTGTGTCCTTTCTTCAACTTTGAAATCGCTTTCGTAATTCTTAATGGCTTT

Figure S1. Sequence alignment of the VTC1 gene sequence of the Col-0 TAIR database, the vtc1-1, svt2 mutants, and the Ler-0 GenBank database. Horizontal arrows denote 5' respectively 3' flanking regions of the sequence insertion, which is highlighted in grey, in the VTC1 promoter region (between base pairs 1990 and 2273). Upright arrows indicate sequences shared between svt2 and Ler. Arrows pointing down denote sequences shared between svt2 and Col. Arrowheads point to sequences unique to svt2. Highlighted in yellow are the start and stop codons, respectively. Highlighted in green is the vtc1-1 mutation. 


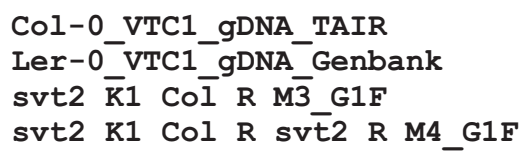

Col-0 VTC1 gDNA TAIR Ler-0_VTC1_gDNA_Genbank svt2 $\overline{\mathrm{K}} 1 \mathrm{Co} \overline{\mathrm{l}} \mathrm{R}$ M $\mathrm{3}$ GIF svt2 K1 Col R svt2 R M4_G1F

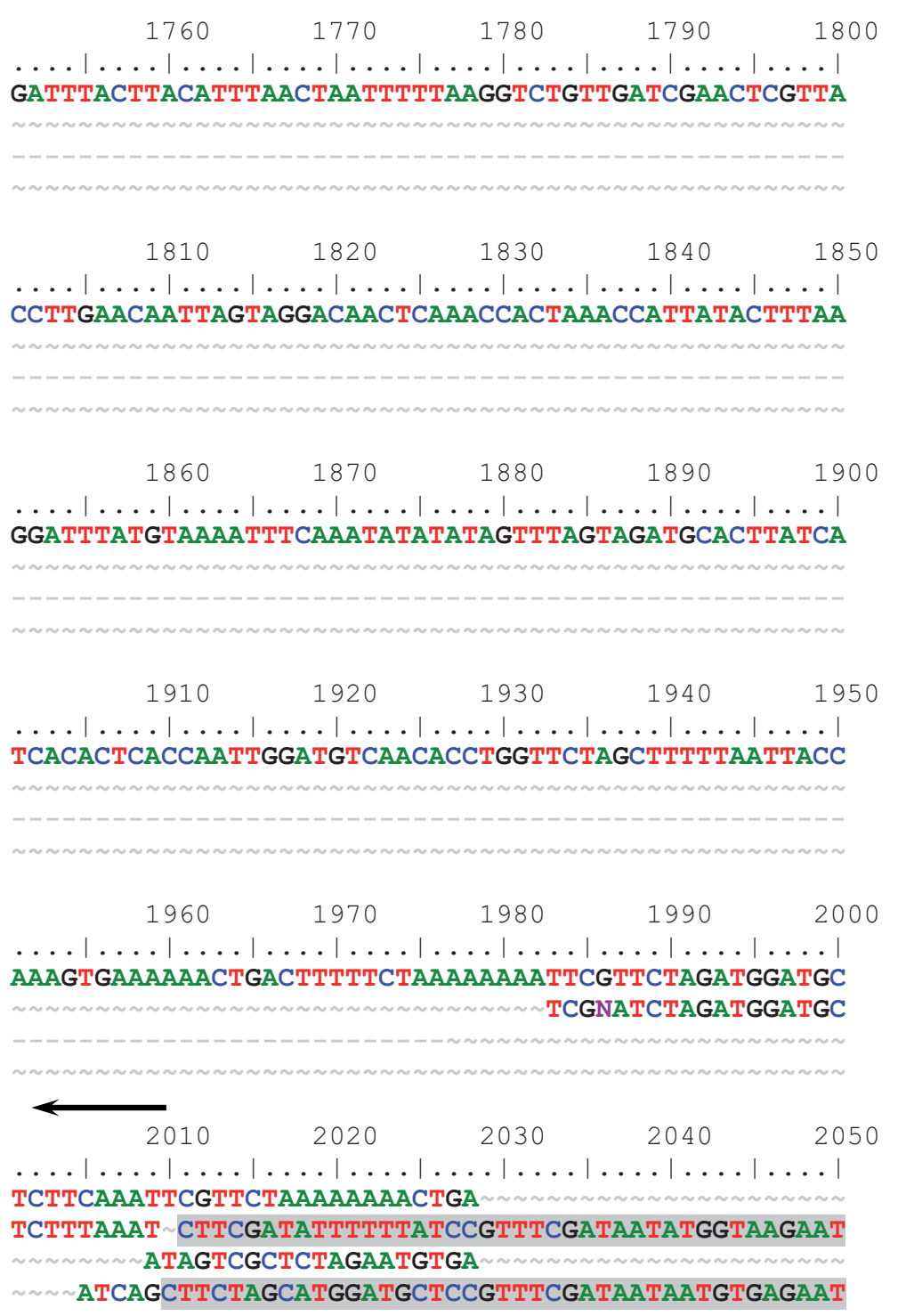

$\ldots+\ldots|\ldots| \ldots|\ldots| \ldots|\ldots| \ldots|\ldots| \ldots|\ldots| \ldots|\ldots| \ldots \mid$

GAATGACGAATCGGTCAAGTAATCTGTATATTAA CATTGTACTCATCA

GAATGACGAATCGGTCAAGCTAATCTGTATATTAATCATTGTACTCATCA

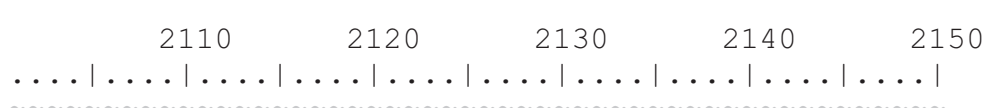

ACGTAAAGTCCTATTCGTCTATACATATGTGAACTTATATATGTCTATCA

ACGTAAAGTCCTATTCGTCTATACATATGTGAACTTATATATGTCTATCA

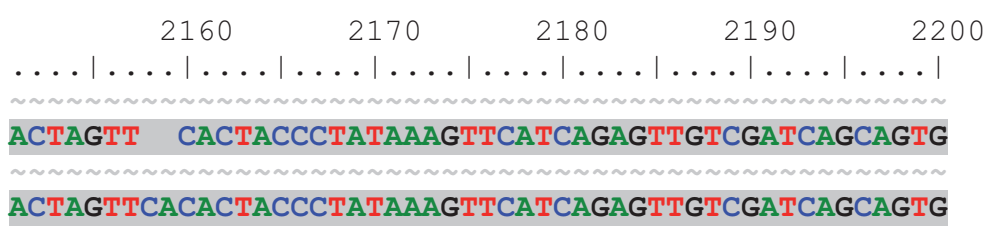


Col-0 VTC1 gDNA TAIR Ler-0_VTC1_gDNA_Genbank svt2 $\overline{\mathrm{K}} 1 \mathrm{Co} \overline{\mathrm{I}} \mathrm{R}$ M $\mathrm{G} 1 \mathrm{~F}$ svt2 K1 Col R svt2 R M4_G1F

COI-0_VTC1_gDNA_TAIR Ler-0 VTC1 gDNA Genbank svt2 $\overline{\mathrm{K}} 1$ Co $\overline{1} \mathrm{R}$ M $\overline{3}$ G1F svt2 K1 Col R svť2 R M4 G1F

Col-0 VTC1 gDNA TAIR Ler-0 VTC1 gDNA Genbank svt2 $\overline{\mathrm{K}} 1 \mathrm{Co} \overline{\mathrm{l}} \mathrm{R}$ M $\overline{3} \mathrm{G} 1 \mathrm{~F}$ svt2 K1 Col $R$ svt2 R M4 G1F

Col-0_VTC1_gDNA_TAIR Ler-0 VTC1 gDNA Genbank svt2 $\overline{\mathrm{K}} 1$ Co $\bar{l} \mathrm{R}$ M $\overline{3}$ G1F svt2 K1 Col $R$ svt̄2 R M4_G1F

Col-0 VTC1 gDNA TAIR Ler-0 VTC1_gDNA Genbank svt2 $\overline{\mathrm{K}} 1 \mathrm{Co} \overline{\mathrm{l}} \mathrm{R}$ M $\mathrm{3} 1 \mathrm{~F}$ svt2 K1 Col R svt2 R M4_G1F

Col-0_VTC1_gDNA_TAIR Ler-0 VTC1 gDNA Genbank svt2 $\overline{\mathrm{K}} 1 \mathrm{Co} \overline{\mathrm{l}} \mathrm{R}$ M $\overline{3} \mathrm{G} 1 \mathrm{~F}$ svt2 K1 Col $R$ svt̄2 R M4_G1F

Col-0 VTC1 gDNA TAIR Ler-0_VTC1_gDNA_Genbank svt2 $\overline{\mathrm{K}} 1$ Co $\bar{l} \mathrm{R}$ M 3 G1F svt2 K1 Col $R$ svt2 $R$ M4_G1F

Col-0_VTC1_gDNA_TAIR Ler-0 VTC1 gDNA Genbank svt2 $\overline{\mathrm{K}} 1$ CoI $\mathrm{R}$ M $\overline{3}$ G1F svt2 K1 Col R svt2 R M4_G1F

COI-0 VTC1 gDNA TAIR Ler-0 VTC1 gDNA Genbank svt2 $\overline{\mathrm{K}} 1 \mathrm{Co} \overline{\mathrm{l}} \mathrm{R}$ M $\overline{3} \mathrm{G} 1 \mathrm{~F}$ svt2 K1 Col R svt2 R M4_G1F

\section{0 \\ 2220 \\ 2230 \\ 2240 \\ 2250 \\ $\ldots|\ldots| \ldots|\ldots| \ldots|\ldots| \ldots|\ldots| \ldots|\ldots| \ldots|\ldots|$ \\ ACCACTACACATTCTTCATACAGCTGAGTTAGGAATGTTAACAAAATAGT \\ ACCACTACACATTCTTCATACAGCTGAGTTAGGAATGTTAACAAAATAGT}

$2260 \quad 2270 \quad 2280 \quad 2290 \quad 2300$

$\ldots|\ldots| \ldots|\ldots| \ldots|\ldots| \ldots|\ldots| \ldots|\ldots| \ldots|\ldots|$

ACATAATACCATCCCTTTAAAAACACAGAAT

TTATGGGAGTATGTTTTATACATAATACCATCCCTTTAAAAACACAGAAT CATAATACCATCCCTTTAAAA-CACAGAAT TTATGGGAGTATGTTTTATACATAATACCATCCCTTTAAAAACACAGAAT

$\begin{array}{lrrr}2310 & 2320 & 2330 & 2340 \\ \cdots & 2350\end{array}$

$\ldots|\ldots| \ldots|\ldots| \ldots|\ldots| \ldots|\ldots| \ldots|\ldots| \ldots|\ldots|$ TTTTTTTATCATCTCTGAAACAAATCATTTACAGTAGTAAATGTCAA--C TTTCTTTATCATCTCCGAAACAAATCATTTACAGTAGTAAATGTAAAAAC TTTTTTTATCATCTCTGAAACAAATCATTTACAGTAGTAAATGTCAA--C TTTCTTTATCATCTCCGAAACAAATCATTTACAGTAGTAAATGTAAAAAC

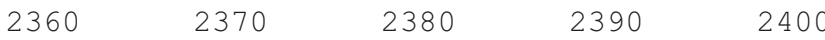
$\ldots|\ldots| \ldots|\ldots| \ldots|\ldots| \ldots|\ldots| \ldots|\ldots| \ldots|\ldots|$ ACAACATTAATTCTGTTT-GTTGTTGGCATTTACAATTGCAAAATCATTT ACAACATTAATTCTGTTT-GTTGTTGGCATTTACAATTGCAAAATCATTT ACAACATTAATTTGGTTTTGTTGTTGGCATTTACAATTGCAAAATCATTT ACAACATTAATTCTGTTT-GTTGTTGGCATTTACAATTGCAAAATCATTT
2410
2420
2430
2440
2450

$\ldots|\ldots| \ldots|\ldots| \ldots|\ldots| \ldots|\ldots| \ldots|\ldots| \ldots|\ldots|$ TCTCATTTATTATTCGTATTTATTTTGTCAAGAACCCTTGTCTCTAAAAT TCTCATTTATTATTCGTATTTATTTTGTCAAGAACCCTTGTCTCTAAAAT TCTCATTTATTATTCGTATTTATTTTGTCAAGAACCCTTGTCTCTAAAAT TCTCATTTATTATTCGTATTTATTTTGTCAAGAACССTTGTCTCTAAAAT

$2460 \quad 2470 \quad 2480 \quad 2490 \quad 2500$ $\ldots|\ldots| \ldots|\ldots| \ldots|\ldots| \ldots|\ldots| \ldots|\ldots| \ldots|\ldots|$ ATTCATAGAAAAAGAAAAGAGCCATTAATTAATGGCTTGAAGAAAGA-TT ATTCATAGAAAAA-AAAAGAGCCATTAATTAATGGCTTGAAGAAAGA-TT ATTCATAGAAAAAGAAAAGAGCCATTAATTAATGGCTTGAAGAAAGA-TT ATTCATAGAAAAA-AAAAGAGCCATTAATTAATGGCTTGAAGAAAGAATT

$\begin{array}{lrrr}2510 & 2520 \quad 2530 & 2540 & 2550\end{array}$ $\ldots|\ldots| \ldots|\ldots| \ldots|\ldots| \ldots|\ldots| \ldots|\ldots| \ldots|\ldots|$ GGTGTATAAGC-GTCTACGTGACCTTTAA--TTAATTTACTT---CCCCC GGTGTATAAGC-GTCTACGTGACCTTTAA--TTAATTTACTT---CCCCA GGTGTATAAGC-GTCTACGTGACCTTTAA--TTAATTTACTT---CCCCC GGTGTATAAACCGTCTACCTGACCTTTAAATTTAATTTACTTTCCCCCCA

$2560 \quad 2570 \quad 2580 \quad 2590 \quad 2600$

$\ldots . \ldots|\ldots| \ldots|\ldots| \ldots|\ldots| \ldots|\ldots| \ldots|\ldots| \ldots \mid$ AAAAAAGTCAAC-ATTCAA-CATGTG-AATAAAAA-TCAATATTGG-TTT AAAAAAGTCAAC-ATTCAA-CATGTG-AATAAAAAATCAATATTGG-TTT AAAAAAGTCAAC-ATTCAA-CATGTG-AATAAAAA-TCAATATTGG-TTT CAAAAAGTCAACCATTCAAACATGGGGAGTAAAAAATCAATATTGGGTTG

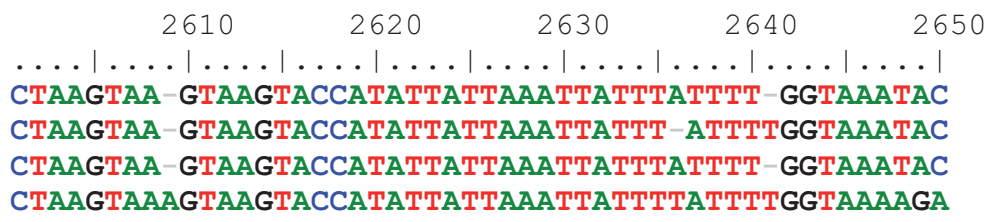


Col-0_VTC1_gDNA_TAIR Ler-0-VTC1_gDNA_Genbank svt2 $\overline{\mathrm{K}} 1$ Co $\overline{1} \mathrm{R}$ M $\overline{3}$ G1F svt2 K1 Col $R$ svt2 R M4_G1F

Col-0_VTC1_gDNA_TAIR Ler-0_VTC1_gDNA_Genbank svt2 $\overline{\mathrm{K}} 1$ Co $\overline{1} \mathrm{R}$ M $\overline{3}$ G1F svt2 K1 Col $R$ svt2 R M4_G1F

Col-0 VTC1 gDNA TAIR Ler-0_VTC1_gDNA_Genbank svt2 $\overline{\mathrm{K}} 1 \mathrm{Co} \overline{\mathrm{l}} \mathrm{R}$ M $\overline{3}$ G1F svt2 K1 Col R svt2 R M4_G1F

Col-0_VTC1_gDNA_TAIR Ler-0_VTC1_gDNA_Genbank svt2 $\overline{\mathrm{K}} 1 \mathrm{Co} \overline{\mathrm{l}} \mathrm{R}$ M $\overline{3} \mathrm{G} 1 \mathrm{~F}$ svt2 K1 Col $R$ svt2 R M4_G1F

Col-0_VTC1_gDNA_TAIR Ler-0 VTC1 gDNA Genbank svt2 $\overline{\mathrm{K}} 1 \mathrm{Co} \overline{1} \mathrm{R}$ M $\overline{3}$ G1F svt2 K1 Col $R$ svt2 R M4_G1F
$2660 \quad 2670 \quad 2680 \quad 2690 \quad 2700$

$\ldots|\ldots| \ldots|\ldots| \ldots|\ldots| \ldots|\ldots| \ldots|\ldots| \ldots|\ldots| \ldots \mid$

-GCACTCAATTTTTCTC--TCAACG----GTGTATATAAACAAAAGGAG

- GCACTCAATTTTTCTC--TCAACGGTGGGTGTATATAAACAAAAGGAG

-GCACTCAATTTTTCTC--TCAACG----GTGTATATAAACAAAAGGAG

СААСАСТСААTTTTTСTCTTCACCGGGGGGGGATGTAAAAAAAAAGAG

$\ldots . \ldots|\ldots| \ldots|\ldots| \ldots|\ldots| \ldots|\ldots| \ldots|\ldots| \ldots \mid$ TCTCСTTTGGAAAAAACTTGCСTATCATTTTGCCAACGAAC-GTTCTTTC TCTCCTTTGGAAAAAACTTGCСTATCATTTTGCCAACGAAC-GTTCTTTC TCTCCTTTGGAAAAAACTTGCCTATCATTTTGCCAACGAAC-GTTCTTTC TCTCСTTTGGAAAAAAAAAAC-TATC-TTTTGCCGCC-AAC-АTTTTTTT

$\begin{array}{llll}2760 & 2770 & 2780 & 2790\end{array} 2800$

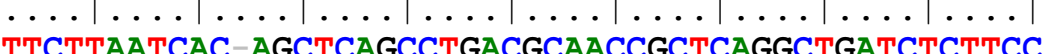
TTCTTAATCAC-AGCTCAGC-СTACGCAACCGCTCAGGCTGATСTCTTCC TTCTTAATCAC-AGCTCAGCCTGACGCAACCGCTCAGGCTGATTGATTCT TCTTTATTCA---GCTCAGC-CTGAGGAACCCCCAGTGGGCATTTTTTAA

\begin{tabular}{c|c|c|c|c|c|c|}
2810 & 2820 & 2830 & 2840 & 2850
\end{tabular}

AАTTTACAGCCATTTCCCAGCTCAGATCTCTGATCCGGTGAGATCTCTCT АATTTACAGCCATTTCCCAGCTCAGATCTCTGATCCGGTGAGATCTCTCT СTTTTTT--TCCCCGAAAAAAAAAAAACAGAAACTGGGGGCAATTTTTTT AAAAG

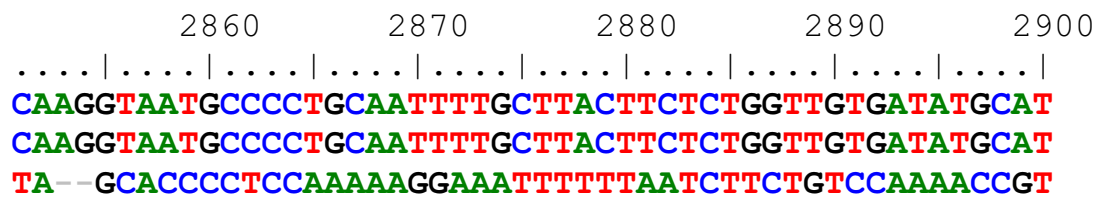

$$
29102920 \quad 2930 \quad 2940 \quad 2950
$$

$\ldots|\ldots| \ldots|\ldots| \ldots|\ldots| \ldots|\ldots| \ldots|\ldots| \ldots|\ldots|$ GTTCTTCGAATTTTCATCGTTTGTGATTTGAATCTCATTTTGTATTTGC GTTCTTCGAATTTTCATCGTTTGTGATTTGAATTCTCATTTTGTATTT-C АTTTTTTTAAGCСССССТTT-

COI-0 VTC1 gDNA TAIR

Ler-0_VTC1_gDNA_Genbank svt2 $\overline{\mathrm{K}} 1 \mathrm{Co} \overline{\mathrm{I}} \mathrm{R}$ M $\overline{3} \mathrm{G} 1 \mathrm{~F}$ svt2 $\mathrm{K} 1$ Col $R$ svt2 R M4 G1F

Figure S2. Sequence alignment of the VTC1 promoter InDel polymorphism sequence of the Col-0 TAIR database, the Ler-0 Genbank database, the svt2 $\mathrm{K} 1 \mathrm{Col} R \mathrm{M}_{3}$ revertant (Col-like phenotype) and the svt2 $\mathrm{K} 1 \mathrm{Col} \mathrm{R}$ svt2 $\mathrm{R} \mathrm{M}_{4}$ revertant (svt2-like phenotype). Horizontal arrows denote 5' respectively 3' flanking regions of the sequence insertion, which is highlighted in grey, in the VTC1 promoter region in Ler-0 and svt2 $\mathrm{K} 1 \mathrm{Col} R$ svt2 R M4, which exhibits an svt2-like phenotype. The svt2 K1 Col R M3 mutant has a Col-like phenotype and share sequence similarities with the Col-O sequence. $R$ denotes revertant. 
1. Conklin PL, Barth C: Ascorbic acid, a familiar small molecule intertwined in the response of plants to ozone, pathogens, and the onset of senescence. Plant Cell Environ. 2004; 27(8): 959-970.

Publisher Full Text

2. Pavet V, Olmos E, Kiddle G, et al.: Ascorbic acid deficiency activates cell death and disease resistance responses in Arabidopsis. Plant Physiol. 2005; 139(3): 1291-1303.

PubMed Abstract | Publisher Full Text | Free Full Text

3. Kotchoni SO, Larrimore KE, Mukherjee M, et al.: Alterations in the endogenous ascorbic acid content affect flowering time in Arabidopsis. Plant Physiol. 2009; 149(2): 803-815.

PubMed Abstract | Publisher Full Text | Free Full Text

4. Mukherjee M, Larrimore KE, Ahmed NJ, et al:: Ascorbic Acid Deficiency in Arabidopsis Induces Constitutive Priming That is Dependent on Hydrogen Peroxide, Salicylic Acid, and the NPR1 Gene. Mol Plant Microbe Interact. 2010; 23(3): 340-351.

PubMed Abstract | Publisher Full Text

5. Arrigoni O, De Tullio MC: The role of ascorbic acid in cell metabolism: between gene-directed functions and unpredictable chemical reactions. $J$ Plant Physio 2000; 157(6): 481-488.

Publisher Full Tex

6. Arrigoni O, De Tullio MC: Ascorbic acid: much more than just an antioxidant. Biochim Biophys Acta. 2002; 1569(1-3): 1-9.

PubMed Abstract | Publisher Full Text

7. Pastori GM, Kiddle G, Antoniw J, et al.: Leaf vitamin C contents modulate plant defense transcripts and regulate genes that control development through hormone signaling. Plant Cell. 2003; 15(4): 939-951.

PubMed Abstract | Publisher Full Text | Free Full Text

8. Qin C, Qian W, Wang W, et al:: GDP-mannose pyrophosphorylase is a genetic determinant of ammonium sensitivity in Arabidopsis thaliana. Proc Natl Acad Sci USA. 2008; 105(47): 18308-18313.

PubMed Abstract | Publisher Full Text | Free Full Text

9. Barth C, Gouzd ZA, Steele HP, et al:: A mutation in GDP-mannose pyrophosphorylase causes conditional hypersensitivity to ammonium, resulting in Arabidopsis root growth inhibition, altered ammonium metabolism, and hormone homeostasis. J Exp Bot. 2010; 61(2): 379-394.

PubMed Abstract | Publisher Full Text | Free Full Text

10. $\mathrm{Li} \mathrm{Q}, \mathrm{Li} \mathrm{BH}, \mathrm{Kronzucker} \mathrm{HJ}$, et al.: Root growth inhibition by $\mathrm{NH}_{4}{ }^{+}$in Arabidopsis is mediated by the root tip and is linked to $\mathrm{NH}_{4}{ }^{+}$efflux and GMPase activity. Plant Cell Environ. 2010; 33(9): 1529-1542.

PubMed Abstract | Publisher Full Text

11. Kempinski CF, Haffar R, Barth $\mathrm{C}$, et al.: Toward the mechanism of $\mathrm{NH}_{4}^{+}$sensitivity mediated by Arabidopsis GDP-mannose pyrophosphorylase. Plant Cell Environ. 2011; 34(5): 847-858

PubMed Abstract | Publisher Full Text

12. Wheeler GL, Jones MA, Smirnoff N, et al:: The biosynthetic pathway of vitamin C in higher plants. Nature. 1998; 393(6683): $365-369$.

PubMed Abstract | Publisher Full Tex

13. Dowdle J, Ishikawa T, Gatzek S, et al: Two genes in Arabidopsis thaliana encoding GDP-L-galactose phosphorylase are required for ascorbate biosynthesis and seedling viability. Plant J. 2007; 52(4): 673-689. PubMed Abstract | Publisher Full Text

14. Conklin PL, Williams EH, Last RL, et al: Environmental stress sensitivity of an ascorbic acid-deficient Arabidopsis mutant. Proc Natl Acad Sci U S A. 1996; 93(18): 9970-9974.

PubMed Abstract | Free Full Text

15. Conklin PL, Norris SR, Wheeler GL, et al: Genetic evidence for the role of GDPmannose in plant ascorbic acid (vitamin C) biosynthesis. Proc Natl Acad SCi U S A. 1999; 96(7): 4198-4203.

PubMed Abstract | Publisher Full Text | Free Full Text

16. Yi H, Richards EJ: Phenotypic instability of Arabidopsis alleles affecting a disease Resistance gene cluster. BMC Plant Biol. 2008; 8: 36 PubMed Abstract | Publisher Full Text | Free Full Text

17. Yi H, Richards EJ: Gene duplication and hypermutation of the pathogen Resistance gene SNC1 in the Arabidopsis bal variant. Genetics. 2009; 183(4): 1227-1234. PubMed Abstract | Publisher Full Text | Free Full Text

18. Weigel D, Glazebrook J: Arabidopsis: A Laboratory Manual. Cold Spring Harbor: Cold Spring Harbor Laboratory Press. 2002, 354.

Reference Source
19. Conklin PL, Saracco SA, Norris SR, et al:: Identification of ascorbic acid-deficient Arabidopsis thaliana mutants. Genetics. 2000; 154(2): 847-856.

PubMed Abstract | Free Full Text

20. De Preter K, Speleman F, Combaret V, et al:: Quantification of MYCN, DDX1, and NAG gene copy number in neuroblastoma using a real-time quantitative PCR assay. Mod Pathol. 2002; 15(2): 159-166.

PubMed Abstract | Publisher Full Text

21. Duarte JM, Wall PK, Edger PP, et al: Identification of shared single copy nuclea genes in Arabidopsis, Populus, Vitis and Oryza and their phylogenetic utility across various taxonomic levels. BMC Evol Biol. 2010; 10: 61.

PubMed Abstract | Publisher Full Text | Free Full Text

22. Molinier J, Ries G, Zipfel C, et al:: Transgeneration memory of stress in plants. Nature. 2006; 442(7106): 1046-1049.

PubMed Abstract | Publisher Full Text

23. Jablonka E, Raz G: Transgenerational epigenetic inheritance: prevalence, mechanisms, and implications for the study of heredity and evolution. Q Rev Biol. 2009; 84(2): 131-176. PubMed Abstract | Publisher Full Text

24. Wang $\mathrm{Y}$, Jha AK, Chen $\mathrm{R}$, et al:: Polyploidy-associated genomic instability in Arabidopsis thaliana. Genesis. 2010; 48(4): 254-263. PubMed Abstract | Publisher Full Text

25. Ranney TG: Polyploid: From Evolution to New Plant Development. Combined Proceedings International Plant Propagators' Society. 2006; 56: 137-142. Reference Source

26. Soltis DE, Soltis PS: The dynamic nature of polyploid genomes. Proc Natl Acad Sci U S A. 1995; 92(18): 8089-8091. PubMed Abstract | Publisher Full Text | Free Full Text

27. Comai L, Tyagi AP, Winter K, et al:: Phenotypic instability and rapid gene silencing in newly formed Arabidopsis allotetraploids. Plant Cell. 2000; 12(9): 1551-1568. PubMed Abstract | Publisher Full Text | Free Full Text

28. Schranz ME, Osborn TC: Novel flowering time variation in the resynthesized polyploid Brassica napus. J Hered. 2000; 91(3): 242-246. PubMed Abstract | Publisher Full Text

29. Ruffio-Chable V, Chatelet $\mathrm{P}$, Thomas $\mathrm{G}$, et al:: Developmentally "aberrant" plants in F1 hybrids of Brassica oleracea. Acta Hort. 2000; 539: 89-94. Reference Source

30. Filkowski J, Kovalchuk O, Kovalchuk I, et al:: Genome stability of vtc1, tt4, and tt5 Arabidopsis thaliana mutants impaired in protection against oxidative stress. Plant J. 2004; 38(1): 60-69. r

PubMed Abstract | Publisher Full Text

31. Roth EJ, Frazier BL, Apuya NR, et al:: Genetic variation in an inbred plant: variation in tissue cultures of soybean [Glycine max (L.) Merrill]. Genetics. 1989; 121(2): 359-368

PubMed Abstract | Free Full Text

32. Chen $\mathrm{Y}$, Lowenfeld $\mathrm{R}$, Cullis $\mathrm{CA}$, et al:: An environmentally induced adaptive (?) insertion event in flax. Int J Biochem Mol Biol. 2009; 1(3): 038-047. Reference Source

33. Lankenau DH, Volff JN: Transposons and the Dynamic Genome. Berlin, Heidelberg: Springer. 2009, 200 Reference Source

34. Lukowitz W, Nickle TC, Meinke DW, et al.: Arabidopsis cyt1 mutants are deficient in a mannose-1-phosphate guanylyltransferase and point to a requirement of $\mathrm{N}$-linked glycosylation for cellulose biosynthesis. Proc Natl Acad Sci U S A. 2001; 98(5): 2262-2267.

PubMed Abstract | Publisher Full Text | Free Full Text

35. Li J, Last RL: The Arabidopsis thaliana trp5 mutant has a feedback-resistant anthranilate synthase and elevated soluble tryptophan. Plant Physiol. 1996; 110(1): 51-59.

PubMed Abstract | Publisher Full Text | Free Full Text

36. Kreps JA, Ponappa T, Dong W, et al:: Molecular basis of alpha-methyltryptophan resistance in amt-1 a mutant of Arabidopsis thaliana with altered tryptophan metabolism. Plant Physiol. 1996; 110(4): 1159-1165. PubMed Abstract | Publisher Full Text | Free Full Text

37. Lolle SJ, Victor JL, Young JM, et al:: Genome-wide non-mendelian inheritance of extra-genomic information in Arabidopsis. Nature. 2005; 434(7032): 505-509. PubMed Abstract | Publisher Full Text

38. Xu PZ, Yuan S, Li Y, et al:: Genome-wide high-frequency non-Mendelian loss of heterozygosity in rice. Genome. 2007; 50(3): 297-302.

PubMed Abstract | Publisher Full Text 


\section{Open Peer Review}

\section{Current Peer Review Status:}

\section{Version 1}

Reviewer Report 31 January 2013

https://doi.org/10.5256/f1000research.866.r747

(C) 2013 Oppenheimer D. This is an open access peer review report distributed under the terms of the Creative Commons Attribution License, which permits unrestricted use, distribution, and reproduction in any medium, provided the original work is properly cited.

\section{David Oppenheimer}

Department of Botany, University of Florida, Gainesville, FL, USA

In this paper, the authors characterize a suppressor of the vtc1-1 mutation, which they named svt2. The authors characterize the phenotype of the vtc1-1 mutants that carry the suppressor mutation and show that the plants have characteristics reminiscent of the Ler accession. Molecular characterization of the suppressed plants show that the suppressor mutation is apparently a reversion of the original vtc1-1 mutation, and surprisingly, have additional genomic signatures of the Ler ecotype as well as additional mutations.

There are several problems with the experimental methods used in this manuscript.

First, according to Figure 1 of the manuscript, the authors screened the $M_{0}$ generation (the mutagenized seed) for wt ascorbic acid content. This is a significant problem for the subsequent mutant analysis in this manuscript. When Arabidopsis seed are mutagenized, the individual cells of the meristem on the seed are mutagenized independently. When the seed germinate, the plants are genetic mosaics. In addition, only those mutations in the L2 layer that gives rise to the germ cells will pass on the mutations. Therefore, it is highly unlikely that a seedling with wt ascorbic acid levels would be isolated from the $\mathrm{M}_{0}$, because it would take the accumulation of many independent mutations, each of which would need to lead to suppression of vtc1.

It is possible that a large sector of an $\mathrm{M}_{0}$ seedling could contain a suppressor mutation that leads to wt ascorbic acid levels, but this sector would have to include cells in the L2 layer for the mutation to be passed on to the next generation. Also, an Ler-like sector should be obvious on a mostly Col-0 plant. Nonetheless, a sector that included the L2 would lead to segregation of the phenotype in the $M_{1}$ generation, because the cells in the sector would be heterozygous for the suppressor mutation.

Second, the concentration of EMS commonly used for mutagenesis in Arabidopsis (0.2\%) is known to cause multiple mutations per genome. When one isolates a mutant of interest from an EMS screen, one should back-cross it at least once to allow these other mutations to segregate away. Otherwise, one may observe unexpected results when analyzing the mutant of interest due to the 
effects of these other mutations.

Third, when analyzing the sequence of the vtc1 gene in the original mutant and in the suppressor, svt2, the authors compared the sequence to the Ler and Col-0 sequences reported in Genbank and TAIR. Instead, the authors should sequence the vtc 1 gene from their original vtc1-1 stock and the $L$ er accession that is present in their lab. This is because it is known that nucleotide polymorphisms arise regularly in lab stocks such that a comparison between a lab stock of Col-0 and the reference sequence can show many differences. Because the authors are reporting unexpected sequencing results, they should show the actual sequence traces (from both strands) for the individual base pair differences highlighted in Figure S1. It would be appropriate to show these sequence traces in the supplemental data. Showing the sequencing traces for the base pair differences would demonstrate that the sequence differences are not due to ambiguous base calling or other sequencing errors.

Fourth, the authors refer to the suppressed plants and their revertants has having a Ler-like or Col-like phenotypes. Because the phenotype of vtc 1 is lower ascorbic acid levels, and the putative suppressor has wt ascorbic acid levels, the ascorbic acid levels in the revertants should be measured to show that they are revertants, instead of relying on the Ler or Col phenotype.

Fifth, because seed and pollen contamination can explain the results, the authors need to explicitly state the degrees to which they tried to eliminate these possible sources of contamination. Were plants of more than 1 genotype grown together? Were seeds of more than 1 genotype collected in the same room? Was soil stored where plants were setting seed? etc.

The single, Ler-like seedling found in the $M_{0}$ population can be explained as an Ler seed that contaminated the Col-0 vtc1-1 seed stock used for the mutagenesis. This can be tested by sowing several thousand Col-0 vtc1-1 seed from that seed stock, and screening them for the presence of any Ler contaminants.

The results in Table 4 are the same as one would expect from seed contamination: the svt2 plants (with the Ler phenotype) have all Ler markers, and the revertants (with the Col phenotype) have all Col markers. The svt2 Col R1 $\mathrm{M}_{2}$ plant highlighted in red shows the expected results if the parent of that plant was heterozygous for Col/Ler. Again, the authors should state what extraordinary measures they used to eliminate seed and pollen contamination.

Once these comments are addressed, the other unexpected results can be examined in a new light.

Competing Interests: No competing interests were disclosed.

I confirm that I have read this submission and believe that I have an appropriate level of expertise to state that I do not consider it to be of an acceptable scientific standard, for reasons outlined above.

Reviewer Report 31 January 2013 


\section{https://doi.org/10.5256/f1000research.866.r744}

(C) 2013 Kovalchuk I. This is an open access peer review report distributed under the terms of the Creative Commons Attribution License, which permits unrestricted use, distribution, and reproduction in any medium, provided the original work is properly cited.

\section{Igor Kovalchuk}

University of Lethbridge, Lethbridge, AB, Canada

Very unusual story. I am still puzzled how this is all possible. I can assume that original vtc1 line had some Ler-1 background (may be from backcrosses). In this case it is possible that the seeds you started with for mutagenesis are highly heterogeneous and some have Ler genomes still present. Now, such a severe case of rearrangements due to combination of EMS and vtc1 background is unbelievable. I wonder why other plants with even greater instability, such as ddm1 or msh2, have never had anything like this reported. Maybe they have not looked for it hard enough? It would make sense to get the vtc1 mutant into rdr2 or rdr6 background (or both) and see whether this RNA cache plays any role - I would expect much lower chance of getting those revertants, same with reverse transcription mutants.

I understand that the event is rare - a single plant was produced - but it would really make the entire story stronger if several different plants were produced.

Competing Interests: No competing interests were disclosed.

\section{I confirm that I have read this submission and believe that I have an appropriate level of expertise to confirm that it is of an acceptable scientific standard.}

Reviewer Report 25 January 2013

https://doi.org/10.5256/f1000research.866.r730

(C) 2013 Pereira A. This is an open access peer review report distributed under the terms of the Creative Commons Attribution License, which permits unrestricted use, distribution, and reproduction in any medium, provided the original work is properly cited.

\section{Andy Pereira}

Departments of Crop, Soil and Environmental Sciences, and Plant Pathology, University of Arkansas, Fayetteville, AR, USA

Very interesting experimental evidence of an inheritance phenomenon that is non-Mendelian and supports an RNA cache hypothesis. The data support the conclusions drawn, but some alternative explanations are put forth that can be addressed.

The EMS mutagenesis treatment of the vtc1-1 genotype yields a single suppressor svt2 mutant that turns out to be a revertant of the vtc1-1 mutation, and is homozygous. Since this screen was from 
1200 seed, it would be interesting to know if such revertant suppressor mutations are also be present in the original batch of vtc1-1 seed used for mutagenesis. Of course since the screen entails a tedious test of TTB on leaves of individual plants for AA content, it is not a recommended control test that should be done, but mechanistically the question remains if the locus is mutable without mutagenesis. What is curious is that the phenotype of the suppressor plant shows a Ler 'plant type' phenotype.

Looking back at the history of the vtc1 mutant, the only time when the Ler and Col genomes were together, described in Conklin et al (1996), was when the vtc1 mutant was crossed to Ler for mapping. The description of the vtc1-1 (soz1) mutant stock (in TAIR) is given as result of $2 \mathrm{x}$ backcrosses and an F3, presumably as a result of crossing to Col-0, but it might be useful to confirm that the stock has no Ler background and the seed used was progeny of single plant and not from a bulk seed lot. Since the VTC1 locus has also been characterized by cyt1 and emb101 mutants, it would seem that the mutant alleles might have some disadvantage in being propagated and a 'residual heterozygosity' might persist by some mechanism. In addition, reversion to a wild-type phenotype svt2 might be facilitated by a selection of vigorous embryos into maturity.

The sequence changes in the vtc1-1 and svt2 suggests an origin of a 'template' independent of Ler and Col alleles, and might also be sequences from another related ecotype. A screen of available Arabidopsis ecotype genome sequences should show such an alternate donor.

Minor comment: On the PDF, page 10, need to use 'were' instead of 'where' in the sentence beginning "In all cases..."

Competing Interests: No competing interests were disclosed.

I confirm that I have read this submission and believe that I have an appropriate level of expertise to confirm that it is of an acceptable scientific standard.

The benefits of publishing with F1000Research:

- Your article is published within days, with no editorial bias

- You can publish traditional articles, null/negative results, case reports, data notes and more

- The peer review process is transparent and collaborative

- Your article is indexed in PubMed after passing peer review

- Dedicated customer support at every stage

For pre-submission enquiries, contact research@f1000.com

F1000Research 